Georgia State University

ScholarWorks @ Georgia State University

8-8-2017

\title{
Cognitive Reappraisal Moderates the Quadratic Association between Heart Rate Variability And Negative Affectivity
}

Jackson M. Gray

Georgia State University

Follow this and additional works at: https://scholarworks.gsu.edu/psych_theses

\section{Recommended Citation}

Gray, Jackson M., "Cognitive Reappraisal Moderates the Quadratic Association between Heart Rate Variability And Negative Affectivity." Thesis, Georgia State University, 2017.

doi: https://doi.org/10.57709/10458175

This Thesis is brought to you for free and open access by the Department of Psychology at ScholarWorks @ Georgia State University. It has been accepted for inclusion in Psychology Theses by an authorized administrator of ScholarWorks@Georgia State University. For more information, please contact scholarworks@gsu.edu. 


\title{
COGNITIVE REAPPRAISAL MODERATES THE QUADRATIC ASSOCIATION \\ BETWEEN HEART RATE VARIABILITY AND NEGATIVE AFFECTIVITY
}

by

\section{JACKSON GRAY}

Under the Direction of Erin C. Tully, Ph.D.

\begin{abstract}
There is uncertainty as to how heart rate variability (HRV), an index of flexible physiological reactivity, relates to an individual's tendency to experience negative emotions. Research suggests that high and low HRV may reflect maladaptive physiological processes associated with negative affectivity and that association may depend on an individual's use of emotion regulation strategies, such as cognitive reappraisal. The current study examined the moderating role of cognitive reappraisal in the quadratic association between HRV and negative affectivity. Electrocardiograms were recorded at rest for 269 young adults who then completed self-report rating scales assessing trait negative affectivity and trait cognitive reappraisal. As predicted, high and low HRV were associated with high negative affectivity at low levels of cognitive reappraisal. These results suggest that, contrary to traditional views, high HRV may not be an adaptive characteristic in the context of low trait reappraisal.
\end{abstract}

INDEX WORDS: Heart rate variability, Cognitive reappraisal, Negative affectivity 


\title{
COGNITIVE REAPPRAISAL MODERATES THE QUADRATIC ASSOCIATION BETWEEN HEART RATE VARIABILITY AND NEGATIVE AFFECTIVITY
}

\author{
by
}

\section{JACKSON GRAY}

A Thesis Submitted in Partial Fulfillment of the Requirements for the Degree of Master of Arts

in the College of Arts and Sciences

Georgia State University 
Copyright by

Jackson Michael Gray 2017 


\section{COGNITIVE REAPPRAISAL MODERATES THE QUADRATIC ASSOCIATION BETWEEN HEART RATE VARIABILITY AND NEGATIVE AFFECTIVITY}

\section{JACKSON GRAY}

Committee Chair: Erin Tully

Committee: Robert Latzman

Aki Masuda

Electronic Version Approved:

Office of Graduate Studies

College of Arts and Sciences

Georgia State University

August 2017 


\section{DEDICATION}

I would like to extend my appreciation to my family, especially my loving parents, who have unconditionally supported me during my years in graduate school. I would also like to thank my friends for being a constant source of support, humor, and relief during stressful times and throughout my graduate school journey. 


\section{ACKNOWLEDGEMENTS}

I would like to acknowledge all of my personal and professional mentors throughout my development that have helped me get to where I am today. Most notably, I would like to thank my advisor, Dr. Erin Tully, for her patience and guidance throughout this journey. It is through her support and encouragement that this project was possible. . I would also like to thank my committee members, Drs. Robert Latzman and Aki Masuda for their invaluable feedback and guidance on this project. 


\section{TABLE OF CONTENTS}

ACKNOWLEDGEMENTS V

LIST OF TABLES

VIII

LIST OF FIGURES IX

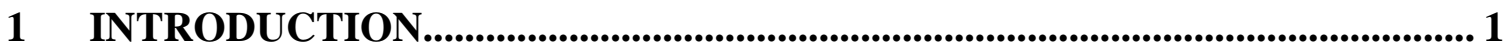

1.1 HRV and the Physiology of Emotion ...................................................... 1

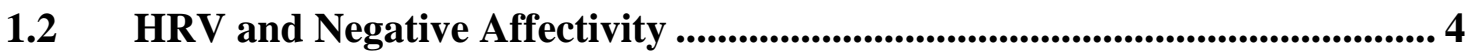

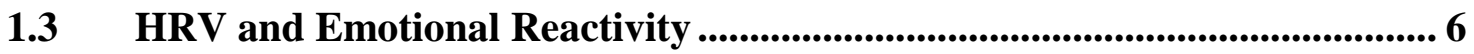

1.4 HRV, Emotion Reactivity, and Negative Affectivity ................................. 8

1.5 Cognitive Reappraisal ...................................................................................... 9

1.6 Purpose and Hypotheses......................................................................... 14

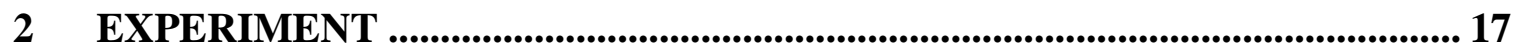

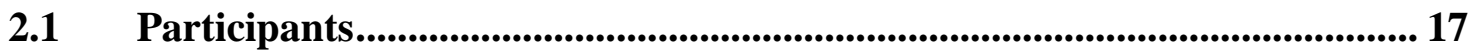

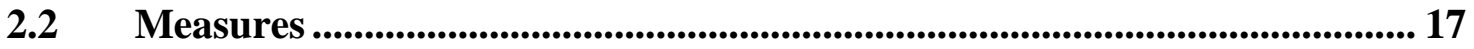

2.2.1 Heart Rate Variability ........................................................................... 17

2.2.2 Cognitive Reappraisal ................................................................................. 18

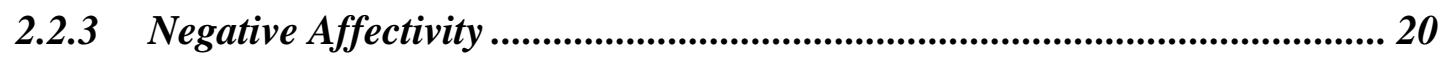

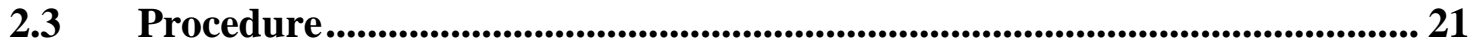

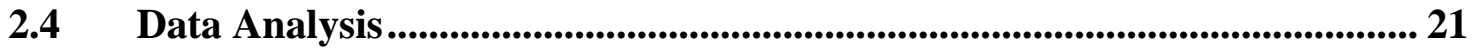

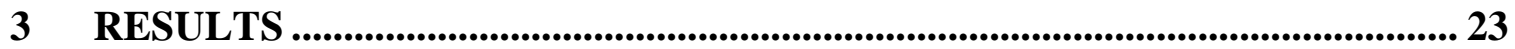


3.1 Descriptive Statistics ................................................................................ 23

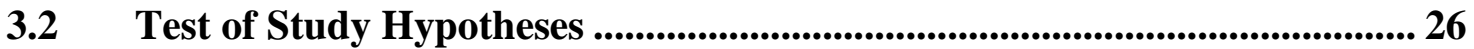

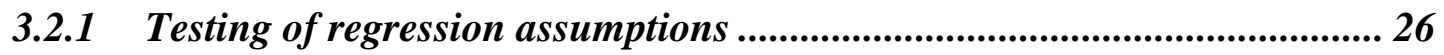

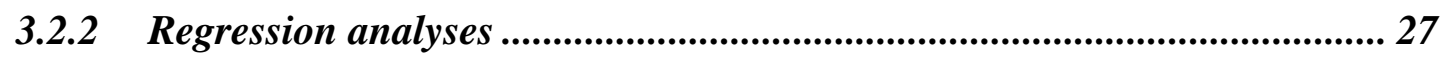

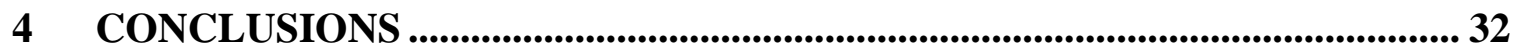

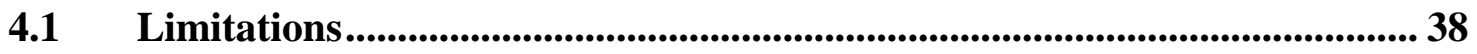

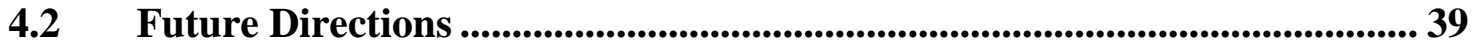

4.3 Conclusions..................................................................................................... 42 


\section{LIST OF TABLES}

Table 1 Means, Standard Deviations, and Zero-Order Correlations between all Continuous

Variables

Table 2 Differences in all continuous variables by gender and race 25

Table 3 Hierarchical curvilinear regression of negative affectivity on heart rate variability moderated by cognitive reappraisal 


\section{LIST OF FIGURES}

Figure 1 Hypothesized quadratic function of HF-HRV on negative affectivity as moderated by cognitive reappraisal. Quadratic effect is only present for low cognitive reappraisal...... 15

Figure 2 Idealized electrocardiograph segment representing two heartbeats. Waveforms are labeled with letters and correspond with specific electrophysiological events during a heartbeat.

Figure 3 Plot of the quadratic association between HF-HRV and negative affectivity moderated by cognitive reappraisal 


\section{INTRODUCTION}

Heart rate variability (HRV) refers to the variations in the durations of beat-to-beat intervals in heart rate. It has received considerable attention as a non-invasive index of flexible physiological reactivity, with HRV in the high frequency domain (HF-HRV) at rest thought to indicate an individual's capacity to generate flexible, regulated physiological responses in emotional contexts (Beauchaine, 2001; Ode, Hilmert, Zielke, \& Robinson, 2010). High HF$\mathrm{HRV}$ is typically conceived as an adaptive characteristic and has been shown to be associated with several aspects of healthy psychological functioning, including high positive emotionality (Oveis et al., 2009), high self-esteem (Schwerdtfeger \& Scheel, 2012) and large attentional capacity (Suess, Porges, \& Plude, 1994). However, investigations of HF-HRV's relation to negative affectivity, which have largely focused on linear associations, have yielded mixed findings, with some studies finding negative, albeit weak, associations (Bliel, Gianaros, Jennings, Flory, \& Manuck, 2008; Riese et al., 2007; Watkins, Grossman, Krishnan, \& Sherwood, 1998) and other studies supporting no association (Oveis et al., 2009; Silvia, Jackson, \& Sopko, 2014; Di Simplicio et al., 2012) and even possible positive associations (Kok \& Fredrickson, 2010). These mixed findings suggest the association between HF-HRV and negative affectivity may more complicated than simple linear associations.

\subsection{HRV and the Physiology of Emotion}

While the exact nature of what constitutes an emotional experience has been the subject of much debate over the past century (Gross, 2014), researchers tend to agree on certain core features of emotions. Emotions are thought to arise when an individual attends to and appraises a situation as being relevant to one of his or her currently active goals (Gross, 1998a, 1998b, 1999, 2014). These appraisals trigger multifaceted responses that involve loosely coupled 
changes in subjective experience, behavior, and, importantly, central and peripheral physiology (Gross, 1998a, 1999, 2014). While it is well established that the emotions humans experience are associated with physiological arousal (Levenson, 2003; Appelhans \& Luecken, 2006), the extent to which individual differences in these physiological processes relate to individual differences in emotion response tendencies are not well understood.

Researchers have proposed empirically-based models to explain the physiological processes associated with emotion experiences (Thayer \& Lane, 2000; Porges, 2007; Porges, Doussard-Roosevelt, \& Maiti, 1994). According to the neurovisceral integration model (NIM), the physiological arousal systems underlying an emotional experience are regulated by a set of interrelated neural structures known as the central autonomic network (CAN; Thayer \& Lane, 2000, 2009). In this model, prefrontal cortical areas like the orbitofrontal cortex (OFC) and medial prefrontal cortex (mPFC) exert top-down inhibitory influences on subcortical limbic structures involved in emotional responding, such as the amygdala (Thayer \& Lane, 2000, 2009; Shekhar, Sajdyk, Gehlert, \& Rainnie, 2003; Barbas, Saha, Rempel-Clower, \& Ghashghaei, 2003). The CAN receives sensory inputs regarding the physiological conditions inside and outside of the body that allow it to dynamically adjust physiological arousal, including the arousal involved in emotional responding, to changing environmental demands (Appelhans \& Luecken, 2006; Thayer \& Lane, 2000). It is important to note that these are implicit processes that are executed outside of the individual's awareness. The primary output of the CAN is mediated through preganglionic sympathetic and parasympathetic neurons, which innervate the heart via the stellate ganglia and vagus nerve, respectively (Thayer \& Lane, 2000). The continuous interplay of these two inputs at the sinoatrial node, the heart's primary pacemaker, produce changes in heart rate (Thayer \& Lane, 2000; 2009; Appelhans \& Luecken, 2006). Thus, 
HRV reflects the continuous interplay between the sympathetic and parasympathetic influences and is directly related to the output of the CAN.

This study will focus on resting HRV, which reflects changes in the moment-to-moment output of the CAN and, by proxy, an individual's capacity to generate regulated physiological responses in the context of emotional responding (Appelhans \& Luecken, 2006; Thayer \& Lane, 2000; Thayer \& Siegle, 2002). Producing an adaptive emotional response depends critically upon the CAN's ability to modulate physiological arousal quickly and flexibly (Appelhans \& Luecken, 2006; Gross, 1998b; Thayer \& Lane, 2000). Due to differences in the response latencies of autonomic neurotransmitters, excitatory sympathetic influences on heart rate are relatively slow compared to inhibitory parasympathetic influences, a phenomenon that allows researchers to quantify the unique variance in heart rate produced by parasympathetic influences in the high frequency (HF) band of HRV (Saul, 1990; Thayer \& Lane, 2000, 2002). Respiratory sinus arrhythmia (RSA), the variability in heart rate linked to breathing, is another commonly used index of parasympathetic influence that is often quantified by HF-HRV (Malliani, Pagani, Lombardi, \& Cerutti, 1991). The physiological arousal systems underlying emotion are under tonic inhibitory via these fast acting inhibitory parasympathetic influences, which allow for the interruption of ongoing behavior and redeployment of resources to other tasks in response to rapid changes in the environment (Berntson et al., 1997; Thayer \& Lane, 2002). These influences also facilitate attending to and appraisal of changes in the internal and external environment and allow the individual to track rapid changes in environmental demands (Thayer \& Lane, 2000, 2002). Thus, the capacity of the CAN to rapidly modulate heart rate via parasympathetic pathways is what allows for flexibility in responding to environmental demands with the varying degrees of physiological arousal that are associated with different emotions 
(Appelhans \& Luecken, 2006). Individual differences in this central and autonomic nervous system integration are indexed by HRV and may be related to individual differences in the tendency to experience certain emotions.

\subsection{HRV and Negative Affectivity}

Low HF-HRV at rest has typically been conceptualized as a trait-level indicator of maladaptive physiological processes that are associated with tendencies to experience negative affect (Oveis et al., 2009; Thayer \& Lane, 2000, 2009). There is some research to support this conceptualization, however the strength of the associations are generally weak (Bliel et al., 2008; Riese et al., 2007; Watkins et al., 1998). Low HF-HRV is thought to reflect poor top-down inhibitory influence over limbic structures involved in emotional responding, which limits the CAN's capacity to modulate physiological arousal quickly and flexibly and thus limits the individual's ability to track rapid changes in environmental demands and organize appropriate responses (Thayer \& Lane, 2000, 2002). This promotes perseveration and continued activation of defensive systems, leading to a reduction in available resources for other processes (Thayer \& Lane, 2002). Individual differences in the functioning of the CAN are likely related to individual differences in tendencies to experience sustained negative emotions. For example, individuals with poor top-down regulation of sympathetic and parasympathetic influences, which can be quantified as low HF-HRV, will be less able to track environmental demands and organize appropriate responses and may become "stuck" in negative emotional states (Thayer \& Lane, 2000; 2002).

Emotional inflexibility and a propensity toward intense negative emotions (e.g., sadness and anxiety) are key characteristics of internalizing pathology. Empirical evidence supports associations between low HF-HRV and internalizing pathology, including major depressive 
disorder (Rottenberg, 2007) and anxiety disorders, such as phobias (Johnsen et al., 2003), panic disorder (Friedman \& Thayer, 1998) and generalized anxiety disorder (Friedman, 2007; Thayer, Friedman, \& Borkovec, 1996). Researchers have used these associations as indirect evidence to support an association between low HF-HRV and high negative affectivity. For instance, Bliel et al. (2008) loaded scores from three well-validated depression and anxiety scales onto a latent "negative affect" variable and found that it had a small-to-moderate size correlation with low HF-HRV. Furthermore, low HF-HRV has been associated with negative perseverative thinking processes, such as rumination (Woody, McGeary, \& Gibb, 2014) and worry (Thayer, Friedman, \& Borkovec, 1996). These perseverative thinking processes are common in depression and anxiety disorders and are moderately-to-highly associated with high negative affectivity (Thomsen, 2006; Mor \& Winquist, 2002). Taken together, these studies support associations between low HF-HRV, emotional inflexibility and negative affectivity.

Research on HF-HRV's relation to negative affectivity and related constructs has yielded mixed findings. Wang, Lu, and Qin (2013) found no association between HF-HRV and negative affectivity as measured by the trait negative affectivity scale of the Positive and Negative Affect Schedule (PANAS; Watson, Clark \& Tellegen, 1988), in a sample of Chinese young adults. Additionally, studies of HF-HRV's relation to neuroticism, a related personality trait that is characterized by the tendency to experience negative emotions, have also yielded mixed findings. Three studies found small-to-moderate sized correlations between low HF-HRV and high neuroticism; the correlation was significant in one of the three studies (Sheperd, Mulgrew, \& Hautus, 2015; Oveis et al., 2009; Ode, Hilmert, Zielke, \& Robinson, 2010). One study found essentially no linear or quadratic associations between HF-HRV and neuroticism; effect sizes were close to zero (Silvia, Jackson, and Sopko, 2014). Another study found evidence 
suggesting a possible positive association between high HF-HRV and high negative affectivity. Kok and Fredrickson (2010) conducted a longitudinal study in which adults had their HRV recorded at an initial assessment session and then rated their experiences of 20 different emotions every day for the next 9 weeks using a modified Differential Emotions Scale (mDES;

Frederickson, Tugade, Waugh, \& Larkin, 2003). They found that adults with higher HF-HRV at the beginning of the study experienced higher levels negative emotions throughout the 9 weeks than those with lower HF-HRV (Kok \& Fredrickson, 2010).

At the state level, Oveis et al. (2009) found no relation between HF-HRV and negative affectivity among healthy young adults using the state version of the PANAS in which individuals reported on their experience of negative emotions over the past month. Similarly, studies have found small nonsignificant differences in HF-HRV for individuals with and without current diagnosis of major depressive disorders in clinical samples (Lehofer et al., 1997; Tulen et al., 1996; Yeragani et al., 1991) and individuals with low versus high levels of current depression symptoms determined via a median split of scores on the Beck Depression Inventory (BDI) in nonclinical samples (Hughes \& Stoney, 2000). One study found that, among currently depressed individuals, there was a small significant positive correlation between high HF-HRV and the sadness symptom on the BDI (Rottenberg, Wilhelm, Gross, \& Gotlib, 2002). Collectively, the empirical evidence for HRV's relation to negative affectivity is weak and inconsistent and has focused almost exclusively on linear relationships, which suggests that the association may be more complex than previously thought.

\subsection{HRV and Emotional Reactivity}

As previously described, HF-HRV appears to index vital aspects of physiological regulation, namely how flexibly the CAN modulates physiological arousal to facilitate regulated 
responses to environmental demands (Friedman \& Thayer, 1998; Thayer \& Lane, 2000). As follows, HF-HRV indicates parasympathetic-sympathetic interplay that allows for tracking changing environmental demands and organizing regulated physiological responses (Thayer \& Lane, 2000). Very high HF-HRV, though, may reflect excessive physiological reactivity as individuals with very high HF-HRV may be prone to heightened attention to and appraisal of changes in the environment, which in turn may lead them to be more emotionally reactive. That is, individuals with very high HF-HRV may be excessively and indiscriminately attending to and appraising changes in the environment to the point that it hinders their ability to focus intently on a specific situation in the face of distraction.

Several lines of research support an association between high HF-HRV and high emotional reactivity. For instance, in a study by Butler et al. (2006), pairs of young adult women had their HF-HRV recorded and were then shown an upsetting film clip about World War II and asked to discuss their thoughts and feelings about it with each other. These researchers found that women with higher HF-HRV experienced and expressed more negative emotions during the conversation that followed the upsetting film clip than those with lower HF-HRV, which suggests that these women may have been more emotionally reactive to the film clip (Butler et al., 2006). In a separate line of research, Ruiz-Padial, Sollers, Vila and Thayer (2003) examined how differences in women's HF-HRV relate to differences in emotion-modulated startle responses when viewing pleasant, neutral, and unpleasant photos. They found that the startle responses of women with the highest HF-HRV were most strongly modulated by the presentation of emotional photographs, which indicates that they were the most emotionally reactive (Ruiz-Padial, Sollers, Vila, \& Thayer, 2003). Similarly, in a study of men responding to the Rorschach test, those with higher levels of HF-HRV reported more positive and negative 
emotions and at greater intensity than those with lower HF-HRV, which suggests that these individuals were more emotionally reactive to the Rorschach stimuli, however these findings need to be interpreted with caution as the Rorschach has been criticized for not having a valid standardized scoring system (Kettunen, Ravaja, Naatanen, \& Keltikangas-Jarvinen, 2000). These studies support the notion that high HF-HRV is associated with high emotional reactivity.

\subsection{HRV, Emotion Reactivity, and Negative Affectivity}

As follows, I propose that the findings that HF-HRV's relation to negative affectivity are weak and inconsistent and may be in part explained by low HF-HRV reflecting deficient topdown regulation of physiological responses to environmental demands that results in high negative affectivity and very high HF-HRV reflecting excessive reactivity to changes in the environment that also results in high negative affectivity. That is, I propose a positive U-shaped quadratic association between HF-HRV and negative affectivity. Geisler, Kubiak, Siewert, \& Weber (2013) found evidence to suggest a possible nonlinear association between HF-HRV and the experience of negative emotions. In this study, HF-HRV was recorded in a sample of young adults who subsequently recorded the number of episodes of sadness and anger they experienced for the next 28 consecutive days using electronic diaries. HF-HRV was significantly higher in participants who reported no anger episodes compared to participants who reported at least one anger episode (Geisler et al., 2013). However, high HF-HRV was associated with a greater number of reported sadness episodes and with the number of reported anger episodes when excluding the participants who reported no anger episodes. Although the authors did not explicitly test for a quadratic association, these results suggest that the association between HFHRV and the experience of negative emotions may be nonlinear. Researchers have found similar nonlinear associations between HF-HRV and aspects of cognitive and social functioning. 
Specifically, very high and very low levels of HF-HRV have been associated with lower prosociality (Kogan et al., 2014) and poorer executive functioning compared to moderate levels of HF-HRV (Spangler, Bell, \& Deater-Deckard, 2015).

However, studies of HF-HRV's nonlinear association with negative affectivity have been limited and have produced mixed findings. Kogan, Gruber, Shallcross, Ford, and Mauss (2013) found a positive quadratic (U-shaped) association between HF-HRV and depression symptoms in healthy adults, such that individuals with both very low and very high HF-HRV had high levels of depression symptoms. Conversely, Silvia et al. (2014) found a negative quadratic (inverse U-shaped) association between HF-HRV and depression symptoms, wherein the opposite was true and individuals with very low and very high HF-HRV had low levels of depression symptoms. Taken together, these studies indicate that extreme levels of HF-HRV are distinct from moderate levels and suggest that the relationship between HF-HRV and negative affectivity may be more complex than previously thought. Additional research testing nonlinear associations and moderators may help elucidate the nature of this association. Recently, researchers have highlighted the need to consider the possible role of emotion regulation strategies in the relation between HF-HRV and emotional responding (Oveis et al., 2009), which may shed some light on these seemingly contradictory findings.

\subsection{Cognitive Reappraisal}

The emotions that humans experience are the result of complex biobehavioral processes that span neural, physiological, and behavioral domains (Porges, 2007; Porges, DoussardRoosevelt, \& Maiti, 1994). Thus, in order to more fully understand individual differences in emotional response tendencies, one must consider not only implicit biological processes but also the more explicit behavioral regulatory processes and how these processes interact to produce the 
emotional responses we experience and observe in one another. Gross' (1998a) process model of emotion regulation illustrates how emotion response tendencies can be influenced by emotion regulation processes at several points in the emotion generation cycle to alter their emotional trajectory. Further, more recent research suggests that individuals' trait-level emotion regulation strategies influence their emotional responding relatively consistently across time and situations (Gross \& John, 2003). As follows, one's general tendency to experience negative emotions is likely the result of the combined influence of their physiological and emotional reactivity tendencies and their use of emotion regulation strategies. Despite this theoretical rationale, to my knowledge no empirical investigations have tested whether an individual's tendency to use certain emotion regulation strategies interacts with his or her capacity for flexible physiological reactivity (i.e., resting HF-HRV) to predict trait negative affectivity. Studying the extent to which linear and quadratic associations between HF-HRV and negative affectivity are affected by trait-level emotion regulation strategies may help explain the complicated, mixed findings about HF-HRV's relation to negative affectivity.

Cognitive reappraisal is one such emotion regulation strategy that conceptually seems like a strategy that might be particularly promising for explaining the mixed findings regarding HF-HRV's relation to negative affectivity. Cognitive reappraisal involves construing potentially emotion-eliciting situations in ways that change their emotional impact (Gross \& John, 2003). It is conceptualized as an antecedent-focused strategy, meaning that it exerts its influence before emotion response tendencies have been fully generated and experienced (Gross \& John, 2003). Research has consistently shown that when used to down-regulate negative emotions, cognitive reappraisal successfully reduces the behavioral experience of negative emotions in the moment (Ray, McRae, Ochsner \& Gross, 2010; Ochsner \& Gross, 2005; Ochsner, Bunge, Gross, \& 
Gabrieli, 2002; Lieberman, Inagaki, Tabibnia, \& Crocket, 2011; Wolgast, Lundh, \& Viborg, 2011; Szasz, Szentagotai, \& Hofmann, 2011; Gross \& John, 2003). Research has also shown that individuals who frequently employ cognitive reappraisal to regulate their emotions experience and tend to express fewer negative emotions (i.e., have lower negative affectivity) than individuals who use the strategy less frequently (Carlson, Dikecligil, Greenberg, \& MujicaParodi, 2012; Dennis, 2007; Ochsner \& Gross, 2005; Wang, Shi, \& Li, 2009; Gross \& John, 2003).

As mentioned previously, emotions involve a complex set of systems (neural, physiological, behavioral) working together in a coordinated fashion in response to some personally relevant event. Researchers have developed models to describe the process of an emotional experience, but these models tend to focus primarily on either biological or behavioral systems underlying an emotional response but not both. For instance, Gross's process model of emotion regulation describes how emotional responses unfold over time and can be modulated from a behavioral standpoint. The NIM, on the other hand, describes the physiological systems underlying an emotional response and how they are regulated by a distributed network of neural systems known as the CAN. These models thus describe different systems that all contribute to the experience of emotions and operate simultaneously.

Recent research on the temporal dynamics and consequences of cognitive reappraisal has illustrated how interrelated these processes are (Goldin, McRae, Rame, \& Gross, 2008). The down-regulation of negative emotions through cognitive reappraisal involves changes in the behavioral, physiological, and neural correlates of negative emotion (Gross, 2002; Gross, 1998a; Ochsner \& Gross, 2005; Gross \& John, 2003; Goldin et al., 2008). In general, studies have found that cognitive reappraisal strategies recruit higher order cognitive control systems, such as 
the dorsal anterior cingulate and PFC regions, to change one's interpretation of a stimulus by modifying levels of activation in emotional arousal-related brain structures, such as the amygdala and insula, thus altering the trajectory of the affective response (Ochsner et al., 2002; Ochsner et al., 2004; Beauregard, Levesque, \& Bourgouin, 2001; Ochsner \& Gross, 2005; Cutuli, 2014; Ochsner \& Gross, 2014). Goldin et al. (2008) found that, relative to participants who were instructed to simply watch a disgust-eliciting film clip, participants who were instructed to reappraise the clip reported experiencing lower levels of negative emotions and exhibited higher early (0-4.5s) activation of prefrontal regions, including the lateral PFC (lPFC), mPFC, and OFC, and activation of the prefrontal regions was associated with significantly lower late (10.5-15s) amygdala and insula activation. Reappraisal has also been shown to reduce peripheral physiological indicators of emotion arousal, for example use of reappraisal has been shown to temper potentiated eyeblink startle responses to negative pictures (Jackson, Malmstadt, Larson, \& Davidson, 2000). Together, these findings suggest that reappraisal strategies intervene early in the emotion-generation cycle, which is evidenced by changes in neural, physiological, and behavioral indices of emotion following cognitive reappraisal, thus highlighting the interconnectedness of these subsystems.

Most of the research on the neural correlates of reappraisal comes from studies in which participants are explicitly instructed to use reappraisal to downregulate responses to emotioneliciting stimuli; however, studies of individual differences in the tendency to use reappraisal have found that similar neural systems are engaged when individuals are spontaneously confronted with negative situations (Drabant, McRae, Manuck, Hariri, \& Gross, 2009). Specifically, Drabrant et al. (2009) found that greater use of reappraisal in everyday life (i.e., higher trait reappraisal) was associated with greater activation of cognitive control regions, such 
as the dIPFC, lateral OFC, and mPFC, and lesser activation in the amygdala when processing emotionally negative facial expressions. While these are still studies of the state-level effects of cognitive reappraisal, I hypothesize that these findings generalize to trait-level. If cognitive reappraisal leads to better top-down regulation of emotion in the moment, it follows that individuals who frequently use reappraisal in everyday life will have better top-down regulation of their emotional responses.

Given the cross-system interconnections between behavioral models of cognitive reappraisal (e.g., Gross \& John, 2003) and models of emotion-related physiological arousal, such as the NIM (Thayer \& Lane, 2000), we can hypothesize about how these two models may be integrated. Low HF-HRV is thought to reflect impairment of the inhibitory mechanisms of the CAN that allow for physiological flexibility (Beauchaine, 2001). Specifically, individuals with low HF-HRV may have reduced capacity for flexible physiological responding (i.e., inflexible CAN) due to low trait-level, top-down inhibitory influences of prefrontal cortical areas over subcortical limbic structures. Cognitive reappraisal involves activation of these inhibitory PFC regions to change one's interpretation of a potentially emotion-eliciting stimulus, ultimately changing the subsequent neural, physiological, and behavioral emotional experience. In the moment, reappraisal increases activity in the inhibitory mechanisms of the CAN and is associated with phasic increases in HF-HRV (Butler et al., 2006; Denson, Grisham, \& Moulds, 2011). Over the long-term, frequent use of reappraisal could lead to enhanced control of emotion by enhancing the integration of the biological and behavioral systems involved in emotional responding. 


\subsection{Purpose and Hypotheses}

In summary, while HF-HRV is typically conceived to have a negative linear association with negative affectivity (Thayer \& Lane, 2000; Thayer et al., 2009), recent theoretical and empirical work suggests that this association may be more complex. I propose that both excessively high and excessively low HF-HRV may reflect maladaptive physiological processes and that the association between HF-HRV and negative affectivity may be influenced by the use of emotion regulation strategies, such as cognitively reappraising negative environmental stimuli to down-regulate the experience of negative emotions.

The purpose of the current study is to examine the moderating role of cognitive reappraisal in the quadratic association between HF-HRV and negative affectivity. I predict both individuals with minimal HF-HRV and individuals with excessive HF-HRV will have elevated levels of negative affectivity but only when they have low cognitive reappraisal tendencies (see Figure 1). Consistent with the NIM, individuals with low HF-HRV are expected to have poorly regulated (i.e., inflexible) physiological reactivity to rapidly changing environmental demands and thus have a greater tendency to experience negative emotions (Thayer \& Lane, 2000; Thayer et al., 2009; Appelhans \& Luecken, 2006), particularly when they are low cognitive reappraisers. At the same time, individuals with very high HF-HRV are expected to have excessively flexible physiological reactivity that leads them be highly emotionally reactive (Butler et al., 2006; RuizPadial et al., 2003), which in the absence of adaptive emotion regulation strategies such as cognitive reappraisal, likely contributes to their tendency to experience negative emotions. According to Gross' (1998) process model of emotion regulation, individuals who frequently use reappraisal to downregulate negative emotion response tendencies before they have been fully generated have lower negative affectivity than individuals who use reappraisal less 


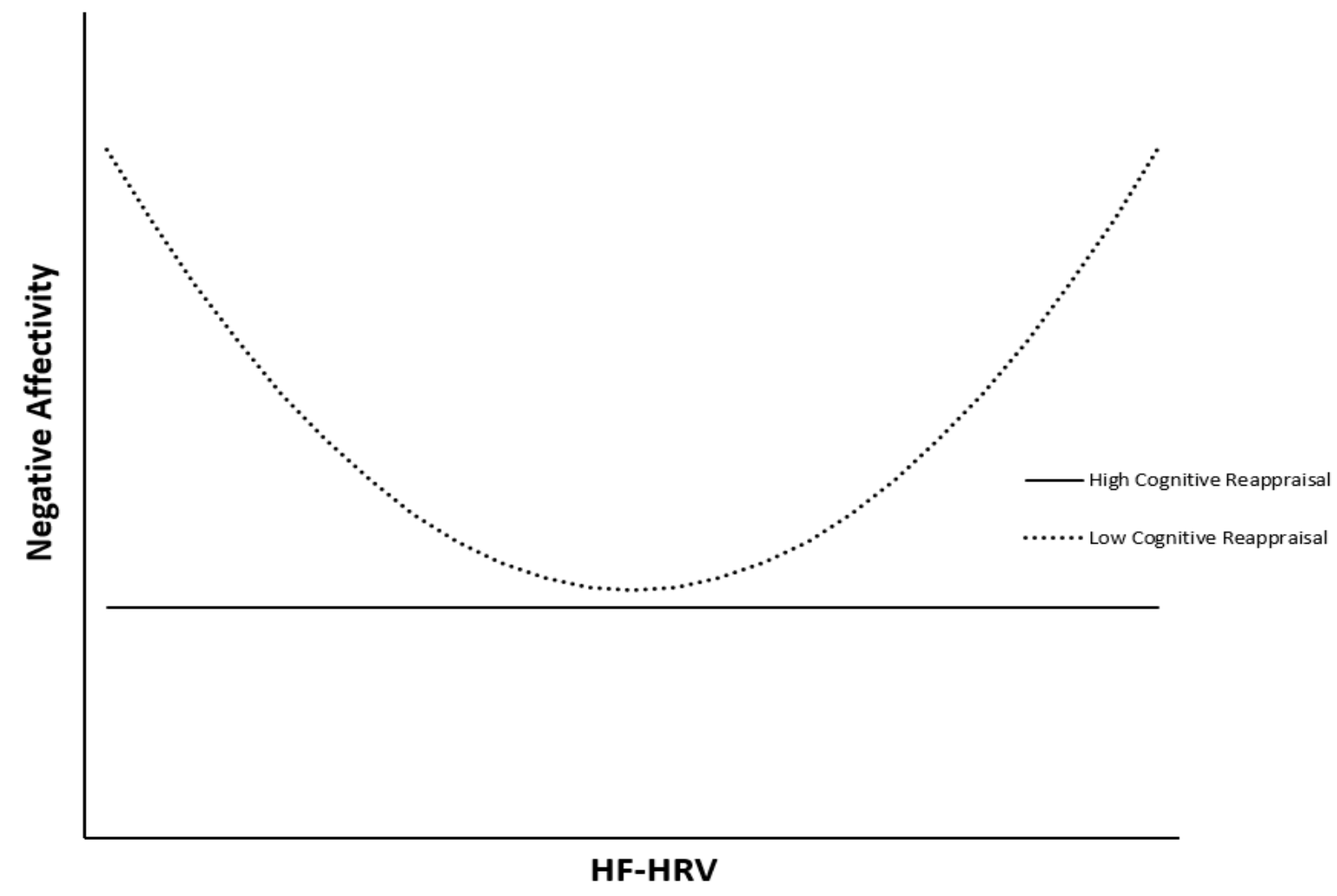

Figure 1 Hypothesized quadratic function of HF-HRV on negative affectivity as moderated by cognitive reappraisal. Quadratic effect is only present for low cognitive reappraisal. 
frequently (Gross \& John, 2003). As follows, I predicted that individuals with high trait reappraisal will have low negative affectivity regardless of their HF-HRV. That is, I hypothesized a positive quadratic association between HF-HRV and negative affectivity and moderation of this quadratic association by cognitive reappraisal such that the quadratic effect will only be present among individuals who tend not to employ cognitive reappraisal strategies to manage their emotional experiences. 


\section{EXPERIMENT}

\subsection{Participants}

Participants were recruited through an online research participant pool of students enrolled in undergraduate psychology courses at a public university in a large city in the southeastern region of the United States in exchange for partial fulfillment of mandatory research credits. The sample consists of 269 young adults ( $78 \%$ female) who ranged in age from 18 to 25 years. The ethnic composition of the sample is representative of the university from which it was drawn, with $43 \%$ self-identifying as "Black/African American", $26 \%$ as "White, not of Hispanic/Latino descent," $11 \%$ as "Asian," $10 \%$ as "Hispanic/Latino," $8 \%$ as "Multiracial," and $2 \%$ as other races. For analyses, participants were grouped into "Blacks/African American," "White, not of Hispanic/Latino descent," and "Other" categories. All participants provided written informed consent prior to the data collection session.

\subsection{Measures}

\subsubsection{Heart Rate Variability}

This study's procedure for recording, scoring, and calculating indices of HRV follow the recommendations of the Task Force of the European Society of Cardiology and the North American Society of Pacing Electrophysiology (1996). Five-minute resting electrocardiograms (ECG) were recorded using three $10-\mathrm{mm} \mathrm{Ag} / \mathrm{Cl}$ disposable electrodes filled with $7 \%$ chloride liquid electrolyte gel. Electrodes were arranged in a standard Lead I formation, with one electrode under the participant's right and left collarbones and one electrode on the outside of his or her left ankle. Raw ECG signals were recorded using the Biopac MP150 data acquisition system and an ECG100C amplifier and were digitized and displayed using AcqKnowledge v4.1 software (Biopac Systems, Inc., Goleta, CA). The signal was sampled at 1,000 Hz and band pass 
filtered between 0.5 and $35 \mathrm{~Hz}$ to improve signal quality. The signal was re-sampled with a cubic-spline interpolation at a resampling frequency of $8 \mathrm{~Hz}$ to generate a continuous timedomain representation of heart rate. AcqKnowledge's peak detection algorithm was used to label the components of each QRS complex and calculate interbeat intervals (IBI: see Figure 2) and HF-HRV. Consistent with Task Force (1996) guidelines, IBIs were defined as the temporal distance between successive R-spikes. IBIs were visually inspected for artifacts (e.g., an unusually long or short R-R interval) and corrected manually by a team of two trained cleaners; a master cleaner and a reliability cleaner. Using percent agreement for identification of R-spikes, initial inter-rater reliabilities were calculated on four five-minute electrocardiograms to ensure adequate training $($ Total IBIs $=1520)$. Both scorers achieved excellent initial inter-rater reliabilities $(\%$ agreements $=99.9 \%, 99.6 \%)$. Following inspection, the cleaned IBIs were then submitted to power spectral analysis in the frequency spectrum of 0.00 to $3.00 \mathrm{~Hz}$. Employing a fast Fourier transformation, power spectral density was quantified in the high frequency (HF; $.15-.40 \mathrm{~Hz}$ ) band in seconds squared. HF power scores were then transformed using the natural logarithm and the resulting HF-HRV values were used in analysis (Kleiger, Stein, \& Bigger, 2005).

\subsubsection{Cognitive Reappraisal}

Participants completed the Emotion Regulation Questionnaire (ERQ; Gross \& John, 2003) to assess their trait-like tendency to engage in two forms of emotion regulation techniques: cognitive reappraisal and expressive suppression. The ERQ is a self-report rating scale comprised of ten statements, six of which measure trait cognitive reappraisal and the other four measure trait expressive suppression. Respondents indicated how they tend to control their emotions by rating items on a 7-point Likert scale ranging from 1 (strongly disagree) to 7 


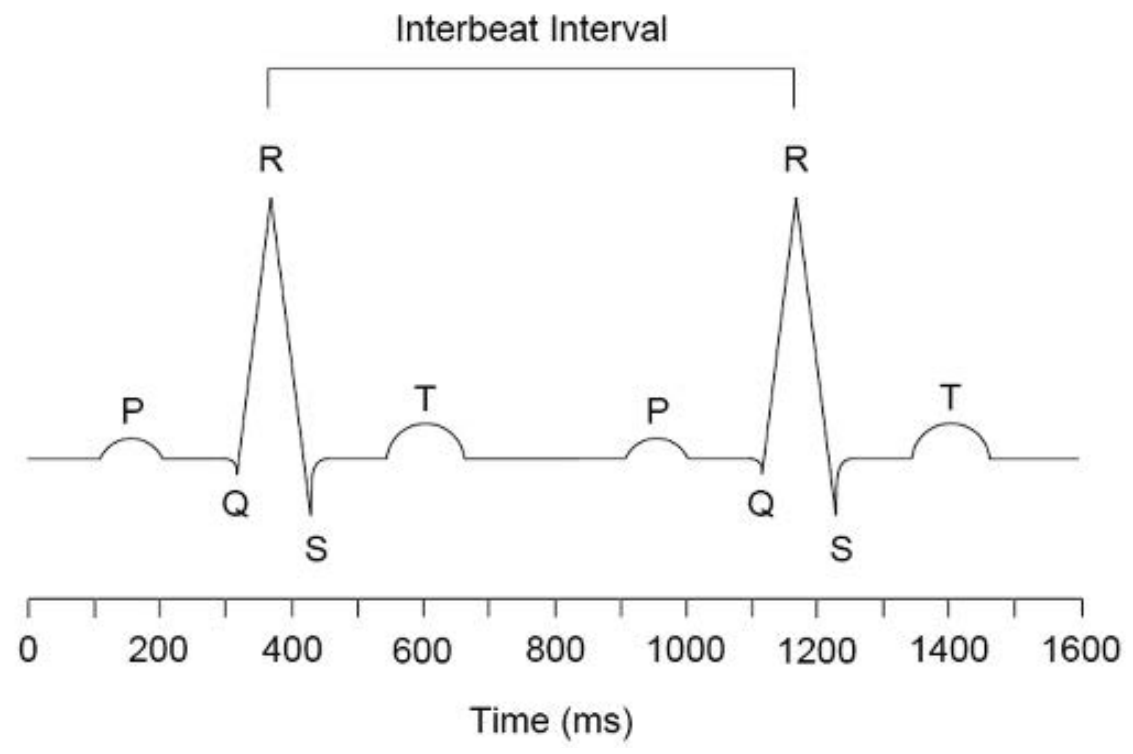

Figure 2 Idealized electrocardiograph segment representing two heartbeats. Waveforms are labeled with letters and correspond with specific electrophysiological events during a heartbeat. The interbeat interval is defined by the temporal distance between successive R-spikes. Reprinted from "Heart Rate Variability as an Index of Regulated Emotional Responding," by B.M. Appelhans and L.J. Luecken, 2006, Review of General Psychology, 10(43), p. 232. Copyright 2006 by the American Psychological Association. 
(strongly agree). For the purposes of the present study I will focus on the trait cognitive reappraisal scale, which measures one's general tendency to modulate their interpretation of potentially emotion-eliciting situations in order to change their emotional impact. This scale includes items such as, "When I want to feel less negative emotion, I change the way I'm thinking about the situation," and "I control my emotions by changing the way I think about the situation I'm in." Items on this scale are summed, with higher scores indicating a greater tendency to use cognitive reappraisal (i.e., trait cognitive reappraisal). The ERQ has strong internal consistency, test-retest reliabilities, and convergent and discriminant validity (Gross \& John, 2003). The internal consistency reliability for the cognitive reappraisal scale was $\alpha=.80$ in our sample.

\subsubsection{Negative Affectivity}

Participants also completed the trait version of the Positive and Negative Affect Schedule - Expanded Form (PANAS-X; Watson \& Clark, 1994), a 60-item measure of specific emotional states, to assess their trait affectivity. The present study used the negative affectivity subscale, which consists of ten items. Participants used a 5-point Likert scale ranging from 1 (very slightly or not at all) to 5 (extremely) to rate the extent to which they generally feel each of the ten negative emotion descriptors (e.g., "irritable" and "upset"). Items are summed such that higher scores indicate a greater tendency to experience negative emotions (i.e., trait negative affectivity). Overall, the PANAS-X has strong psychometric properties, and the trait negative affectivity scale has strong convergent and discriminant validity and internal consistency reliability (Watson \& Clark, 1994; Crawford \& Henry, 2004). The internal consistency reliability for the negative affectivity scale was $\alpha=.84$ in our sample. 


\subsection{Procedure}

The current study was approved by the International Review Board (IRB) and procedures were carried out in accordance with the IRB's standards. Participation entailed one approximately three-hour visit in the FEELINGS Lab at Georgia State University. After informed consent was obtained, the participants completed the PANAS-X, ERQ, and a demographic form that included age, race, and gender, among other demographic variables. They were then fitted with electrodes and instructed to remain seated in a supine position with their eyes open. Following a brief acclimation period, a 5-minute resting ECG recording was obtained while participants sat quietly in a room alone listening to a nature CD, "African Soundscape." In the case that participants moved excessively, closed their eyes, or fell asleep, research assistants corrected this behavior and restarted the rest period. Participants then completed the rest of the study protocol, which included other rating scales, psychophysiological paradigms, and computerized social-cognitive tasks.

\subsection{Data Analysis}

All analyses were performed using SPSS version 23 for Windows (IBM Corp., Armonk, NY, USA). The primary hypothesis was tested using hierarchical multiple nonlinear moderated regression in which the interaction between the quadratic effect of HF-HRV and cognitive reappraisal was used to predict negative affectivity. Prior to running the regression analysis, both HF-HRV and cognitive reappraisal were mean centered. A quadratic term for HF-HRV was computed by squaring the linear HF-HRV term. Interaction terms were computed by multiplying the effects of cognitive reappraisal by the linear and quadratic effects of HF-HRV. Dummy variables were created for the categorical covariates race and gender, with Black/African American and female serving as the reference groups, respectively, as these 
groups have been shown to differ significantly in HF-HRV (Liao et al., 1995; Zahn et al., 2016; Wang, Thayer, Treiber, \& Snieder, 2005; Healy, Treadwell, \& Reagan, 2011). Variables were entered in the hierarchical regression in the following order: Step 1) covariates, including age, race, and gender, Step 2) HF-HRV and cognitive reappraisal, Step 3) the interaction term between HF-HRV and cognitive reappraisal, Step 4) the quadratic term for HF-HRV, and Step 5) the interaction term between the quadratic effect of HF-HRV and the linear effect of cognitive reappraisal. If the HF-HRV ${ }^{2} \mathrm{X}$ Reappraisal interaction effect was deemed significant $(p<.05)$, simple slope analyses were conducted to explicate the interaction. The moderator, cognitive reappraisal, was re-centered at one standard deviation above and below the sample mean. Additional interaction terms were computed by multiplying these newly created moderator variables by the linear and quadratic terms for HF-HRV. Then, two additional hierarchical multiple regressions were conducted to examine the effect of HF-HRV on negative affectivity at high and low levels of cognitive reappraisal. The assumptions of regression were evaluated for all analyses. 


\section{RESULTS}

\subsection{Descriptive Statistics}

Descriptive statistics, including means, standard deviations, and zero-order correlations for all continuous variables are presented in Table 1. An individual's score on a measure was dropped if more than $25 \%$ of the items were skipped, which resulted in two missing cases for the cognitive reappraisal scale. Cognitive reappraisal was significantly negatively correlated to negative affectivity. No other significant correlations were found.

A series of t-tests and ANOVAs were run to test for differences in study variables by race and gender. As shown in Table 2, men and women did not differ significantly in mean levels of HF-HRV, cognitive reappraisal, age, or negative affectivity. As indicated by a one-way ANOVA, there was a main effect of race on HF-HRV. Post hoc comparisons using Hochberg's GT2 criterion indicated that Black/African American participants had significantly higher levels of HF-HRV than participants in the White $(d=0.41, p=.029)$ and Other race $(d=0.45, p=$ .007) groups. Black/African American and White participants did not differ significantly in mean levels of reappraisal $(d=0.02, p=.998)$, negative affectivity $(d=0.30, p=.132)$, or age $(d$ $=0.06, p=.960)$. Black/African American participants also did not differ significantly from Other race participants in mean levels of reappraisal $(d=0.01, p=1.00)$, negative affectivity $(d$ $=0.30, p=.109)$, or age $(d=0.03, p=.994)$. White and Other race participants did not differ significantly in mean levels of HF-HRV $(d=0.43, p=.986)$, reappraisal $(d=0.03, p=.994)$, negative affectivity $(d=0.00, p=1.00)$, or age $(d=0.10, p=.899)$. 
Table 1 Means, Standard Deviations, and Zero-Order Correlations between all Continuous Variables

\begin{tabular}{|c|c|c|c|c|c|c|}
\hline & Mean & $S D$ & 1. & 2. & 3. & 4. \\
\hline 1. HF-HRV & -3.847 & .457 & - & & & \\
\hline 2. Cognitive Reappraisal & 29.464 & 7.096 & .026 & - & & \\
\hline 3. Negative Affectivity & 19.538 & 6.494 & .031 & $-.136^{*}$ & - & \\
\hline 4. Age & 19.680 & 1.702 & -.031 & .018 & .052 & - \\
\hline
\end{tabular}

Note. Logarithmic transformation of HF-HRV is presented. $S D=$ standard deviation; $\mathrm{HF}-\mathrm{HRV}=$ high frequency-heart rate variability. ${ }^{*} p<.05$ 
Table 2 Differences in all continuous variables by gender and race

\begin{tabular}{|c|c|c|c|c|c|c|c|c|c|c|c|c|c|c|c|c|}
\hline & \multicolumn{7}{|c|}{ Gender } & \multicolumn{9}{|c|}{ Race } \\
\hline & \multicolumn{2}{|c|}{ Men } & \multicolumn{2}{|c|}{ Women } & \multirow[b]{2}{*}{$t$} & \multirow[b]{2}{*}{$p$} & \multirow[b]{2}{*}{$d$} & \multicolumn{2}{|c|}{ Black } & \multicolumn{2}{|c|}{ White } & \multicolumn{2}{|c|}{ Other } & \multirow[b]{2}{*}{$F$} & \multirow[b]{2}{*}{$p$} & \multirow[b]{2}{*}{$\eta^{2}$} \\
\hline & $M$ & $S D$ & $M$ & $S D$ & & & & $M$ & $S D$ & $M$ & $S D$ & $M$ & $S D$ & & & \\
\hline HF-HRV & 0.03 & 0.45 & -0.01 & 0.46 & -0.58 & .563 & 0.09 & 0.11 & 0.42 & -0.07 & 0.46 & -0.09 & 0.47 & 5.75 & .004 & 0.04 \\
\hline $\begin{array}{l}\text { Cognitive } \\
\text { Reappraisal }\end{array}$ & -0.08 & 6.61 & 0.18 & 7.24 & 0.25 & .804 & 0.04 & 0.15 & 7.04 & -0.02 & 7.16 & 0.23 & 7.20 & 0.03 & .974 & 0.00 \\
\hline $\begin{array}{l}\text { Negative } \\
\text { Affectivity }\end{array}$ & 1.28 & 0.14 & 1.27 & 0.14 & -0.40 & .692 & 0.07 & 1.24 & 0.14 & 1.29 & 0.14 & 1.29 & 0.14 & 2.96 & .053 & 0.02 \\
\hline Age & 19.90 & 1.69 & 19.62 & 1.70 & -1.11 & .266 & 0.17 & 19.67 & 1.79 & 19.78 & 1.70 & 19.61 & 1.60 & 0.20 & .820 & 0.00 \\
\hline$n$ & 5 & & & & & & & & & & & & & & & \\
\hline
\end{tabular}

Note. $M=$ Mean; $S D=$ standard deviation; HF-HRV = high frequency-heart rate variability. 


\subsection{Test of Study Hypotheses}

\subsubsection{Testing of regression assumptions}

In preparation, univariate descriptive statistics were inspected for data entry errors and regression diagnostics were used to ensure the assumptions of multiple regression were met. As recommended by Tabachnick and Fidell (2013), variables of interest in this study with standardized scores in excess of three standard deviations were flagged as potential univariate outliers and inspected to ensure there was no error in data entry. Two cases were identified as outliers; one cognitive reappraisal and one HF-HRV. These cases were assigned values that were one unit larger (or smaller) than the next most extreme score in the distribution to reduce their impact (Tabachnick \& Fidell, 2013). The assumption of normality was examined using the Kolmorgorov-Smirnov test of normality in conjunction with histograms and Q-Q plots. The distribution of negative affectivity, the outcome variable, was skewed (skewness $=.831, S E=.149$.) and differed significantly from a normal distribution, $D(269)=.109, p<.001$, so a logarithmic transformation of this variable was used in analyses. The distribution of cognitive reappraisal also differed significantly from a normal distribution, $D(269)=.063, p=$ .012. Logarithmic, square root, and reverse score transformations were all tested; however, examination of the histograms and Q-Q plots of each indicated that the original cognitive reappraisal variable was still the most normally distributed and was thus used in analyses. To evaluate the assumption of homoscedasticity of residuals, the standardized predicted values were plotted against the standardized residuals. No discernable patterns that would indicate heteroscedasticity were observed. Multicollinearity was not examined 
for the quadratic interaction term included in the final model as it is necessarily correlated with the linear terms that comprise it and this collinearity typically does not present a problem in nonlinear models (Jaccard \& Turrisi, 2003). Examination of the VIF's for the remaining terms indicated no problem with multicollinearity.

The Mahalanobis distance statistic, a measure of the distance of each case to the centroid of all cases, was computed in order to identify possible multivariate outliers (Tabachnick \& Fidell, 2013). Nine cases were found to have a Mahalanobis distance greater than 27.877 (the critical value associated with $\alpha=.001, d f=9$ ) and were flagged for further examination (Tabachnick \& Fidell, 2013). The Cook's D and DFBeta statistics were then calculated for all of the flagged residuals to determine if they exerted undue influence on the outcome of the regression analysis. Specifically, Cook's D and DFBeta values greater than one indicate that the data point has a large effect on the regression analysis and should be deleted (Stevens, 2002; Cook \& Weisberg, 1982; Bollen \& Jackman, 1990; Belsey, Kuh, \& Welsch, 1980; Tabachnick \& Fidell, 2013). None of the flagged residuals exceeded these values and thus were retained in analyses.

\subsubsection{Regression analyses}

Results from the regression analyses of negative affectivity on the interaction between the quadratic effect of HF-HRV and cognitive reappraisal are displayed in Table 3. The observed power for the hypothesized effect was .966. Of the covariates included in Step 1, the Black/African American race group had significantly lower levels of negative affectivity than the Other race group and nearly significantly lower levels of negative affectivity than the White race group. Age and gender were not significant predictors of negative affectivity. In Step 2, the linear effects of HF-HRV and cognitive 
Table 3 Hierarchical curvilinear regression of negative affectivity on heart rate variability moderated by cognitive reappraisal

\begin{tabular}{|c|c|c|c|c|c|}
\hline Predictors & $\Delta R^{2}$ & $B$ & $S E$ & $\beta$ & $p$ \\
\hline Step 1 & .023 & & & & .181 \\
\hline Gender & & .003 & .021 & .007 & .904 \\
\hline Age & & .003 & .005 & .038 & .536 \\
\hline \multicolumn{6}{|l|}{ Race } \\
\hline Black vs. White & & .041 & .021 & .132 & .051 \\
\hline Black vs. Other & & .042 & .020 & .139 & .039 \\
\hline Step 2 & .022 & & & & .048 \\
\hline HF-HRV & & .021 & .019 & .069 & .263 \\
\hline Reappraisal & & -.003 & .001 & -.136 & .026 \\
\hline Step 3 & .001 & & & & .637 \\
\hline HF-HRV x Reappraisal & & -.001 & .003 & -.029 & .637 \\
\hline Step 4 & .008 & & & & .136 \\
\hline HF-HRV ${ }^{2}$ & & .045 & .030 & .091 & .136 \\
\hline Step 5 & .051 & & & & .000 \\
\hline HF-HRV ${ }^{2} \times$ Reappraisal & & -.017 & .005 & -.284 & .000 \\
\hline
\end{tabular}

Note. Unstandardized and standardized regression coefficients are presented. Gender was represented as a dummy variable with female serving as the reference group. Race was represented as two dummy variables with Black/African American serving as the reference group. $S E=$ standard error; HF-HRV $=$ high frequency-heart rate variability. 
reappraisal were added. Cognitive reappraisal significantly predicted lower levels of negative affectivity above the effects of gender, age, and race, whereas the linear effect of HF-HRV did not. Neither the interaction term between HF-HRV and cognitive reappraisal included in Step 3 nor the quadratic effect of HF-HRV included in Step 4 were significant predictors of negative affectivity. As expected, the interaction between the quadratic effect of HF-HRV and linear effect of cognitive reappraisal included in Step 5 significantly predicted negative affectivity above the effects of gender, age, and race. Tests of simple slopes conducted at one standard deviation above and below the sample mean of cognitive reappraisal revealed that at low levels of cognitive reappraisal, there was a significant positive quadratic association between HF-HRV and negative affectivity, $\beta=.327, p<.001$. The quadratic association between HF-HRV and negative affectivity at high levels of cognitive reappraisal was not significant, $\beta=-.175, p=.056^{1}$. The linear association between HF-HRV and negative affectivity at high levels of cognitive reappraisal was also not significant, $\beta=.038, p=.67$.

Plots of the simple slopes analysis at one standard deviation above and below the sample mean of cognitive reappraisal are displayed in Figure 3. At low levels of trait cognitive reappraisal, there was a negative association between HF-HRV and negative affectivity at low levels of HF-HRV. This negative association between HF-HRV and negative affectivity at low levels of reappraisal diminished with increasing HF-HRV, such that HF-HRV and negative affectivity were not associated at moderate levels of HFHRV. At even higher levels of HF-HRV, the direction of the association reversed

\footnotetext{
${ }^{1}$ When the moderator, cognitive reappraisal, was re-centered at $1.5 \mathrm{SD}$ above the mean, which is within the observed range but at the 93rd percentile, there was a significant negative quadratic association between HF-HRV and negative affectivity, $\beta=-.303, p=.011$, such that, among individuals with extremely high levels of trait reappraisal, very high and low HF-HRV were associated with the lowest levels of negative affectivity.
} 
(became increasingly positive), such that higher levels of HF-HRV were associated with higher levels of negative affectivity. That is, when individuals do not frequently employ cognitive reappraisal as an emotion regulation strategy, those with very low or very high HF-HRV have high levels of negative affectivity. At high levels of trait reappraisal there appeared to be a subtly negative quadratic association between HF-HRV and negative affectivity; however, neither the linear nor the quadratic effect was significant.

In summary, the primary hypothesis was supported; the quadratic effect of HFHRV on negative affectivity was indeed moderated by cognitive reappraisal As predicted, high and low HF-HRV were associated with high negative affectivity at low levels of trait cognitive reappraisal. The quadratic association between HF-HRV and negative affectivity at high levels of trait reappraisal was not significant. 


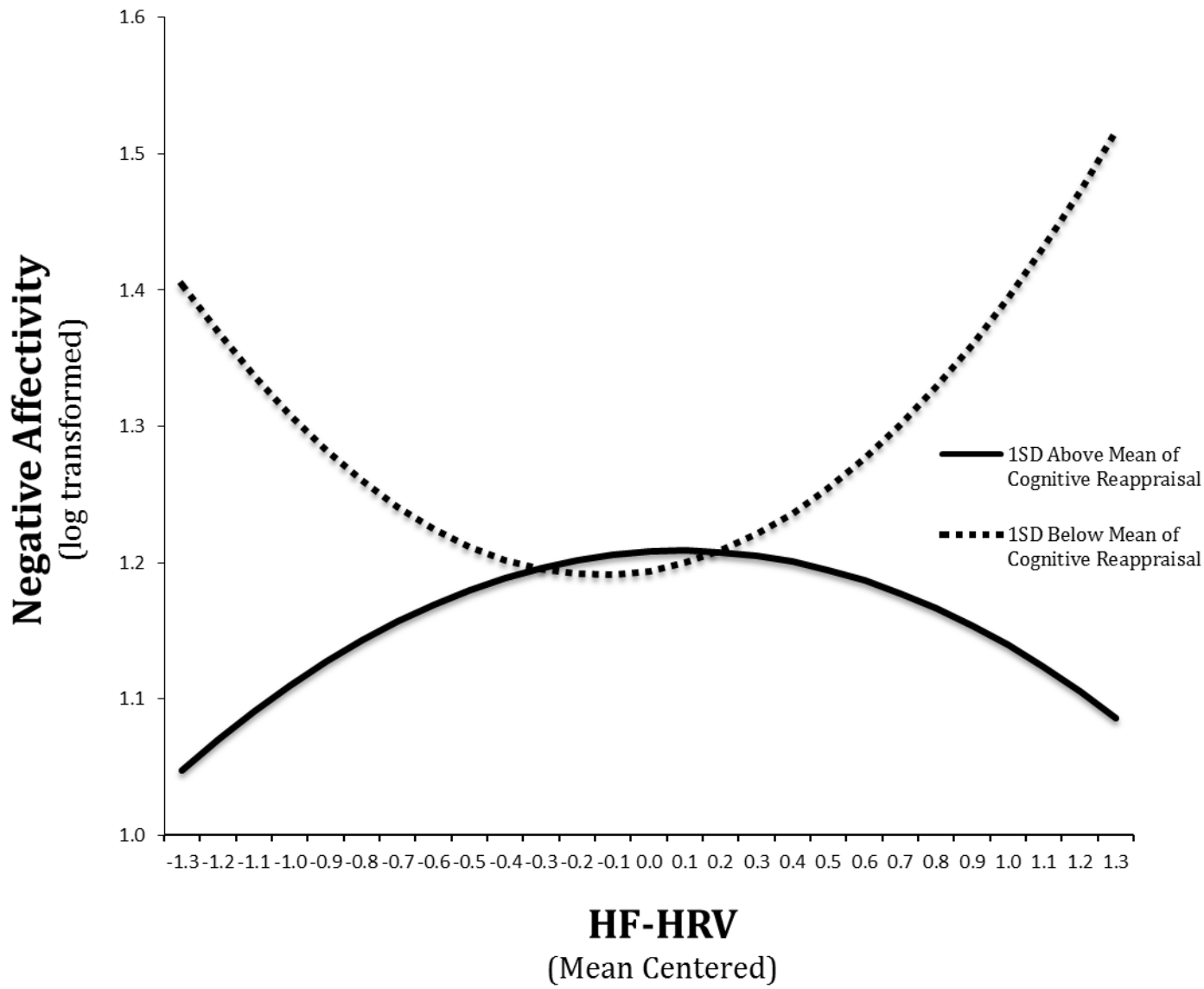

Figure 3 Plot of the quadratic association between HF-HRV and negative affectivity moderated by cognitive reappraisal 


\section{CONCLUSIONS}

Resting HF-HRV is thought to be an index of an individual's capacity for flexible physiological reactivity (Thayer \& Lane, 2000; Beauchaine, 2001). A growing body of theory and research suggests that HF-HRV figures prominently in emotional responding (Appelhans \& Luecken 2006; Butler et al., 2006); however, the extent to which individual differences in the physiological processes indexed by HF-HRV relate to the tendency to experience negative emotions is not well understood. Investigations of HFHRV's relation to negative affectivity have produced mixed results with studies supporting negative (Bliel et al., 2008; Riese et al., 2007; Watkins et al., 1998), positive (Kok \& Fredrickson, 2010; Rottenberg et al., 2002), and no associations between HFHRV and the tendency to experience negative emotions (Silvia et al., 2014). Recent research suggests that the relation between HF-HRV and trait affectivity may be more complex than previously thought; for example the association may be nonlinear and depend upon individuals' trait-like emotion regulation tendencies (Oveis et al., 2009). The central aim of the current study was to ascertain whether very high and low HF-HRV were associated with high negative affectivity and if this association depended on individual differences in one's trait-like tendency to use cognitive reappraisal to regulate their emotions.

Although high HF-HRV is typically conceived of as an adaptive characteristic associated with tendencies to experience low levels of negative emotions (Thayer \& Lane, 2000; Thayer et al., 2009; Appelhans \& Luecken, 2006), results from the current study indicate that this conceptualization is too simplistic. I found individuals with very high HF-HRV and individuals with very low HF-HRV had high trait-like tendencies to 
experience negative emotions but only when they had low cognitive reappraisal tendencies (i.e., tend not to control their emotions by changing the way they think about a situation). In contrast, individuals who frequently use cognitive reappraisal as an emotion regulation strategy had low negative affectivity regardless of their levels of HFHRV. These findings suggest one possible explanation for previous mixed findings regarding HF-HRV's relation to negative affectivity is that the association may be nonlinear and differ as a function of reappraisal tendencies.

Findings from the current study indicate that individuals with low HF-HRV and low reappraisal tendencies exhibit high negative affectivity. As described previously, the physiological arousal systems involved in emotional responding are under tonic inhibitory control via fast-acting parasympathetic influences indexed by HF-HRV (Appelhans \& Luecken, 2006; Berntson et al., 1997). One interpretation of this finding is that, when this inhibitory control is low, as is the case for individuals with low HF-HRV, it may reflect consistent disinhibition of sympathoexcitatory influences, which promote a fight-or-flight response and the continued activation of defensive systems, thereby limiting the individual's ability to track and respond flexibly to changing environmental demands. Ultimately, this can make it difficult for the individual to detect and experience 'safety' when it is in fact present and can lead to irritability, hostility, and anxiety (Thayer \& Lane, 2000; 2002). It is important to note thought that low HF-HRV is only associated with high negative affectivity among individuals with low reappraisal tendencies. These individuals are not changing the way they think about a situation in order to alter its emotional impact and further, in the absence of reappraisal, these individuals may be engaging in other, potentially maladaptive, regulation strategies, such 
as rumination, which could lead the individual to become "stuck" in negative emotional states and contribute to their high negative affectivity (Thayer \& Lane, 2002). This finding is in line with studies indicating that individuals with low HF-HRV have inflexible emotion-modulated startle reactivity (Ruiz-Padial et al., 2003) as well as studies that have found negative associations between HF-HRV and negative affectivity related constructs, like neuroticism (Bliel et al., 2008; Shepherd, Mulgrew, \& Hautus, 2015; Oveis et al., 2009; Ode et al., 2010). Results of the current study extend previous work by showing that this association does exist but is present only in individuals with relatively inflexible physiological reactivity and low trait reappraisal.

This association between HRV and negative affectivity disappeared at sample mean level of HF-HRV, suggesting the maladaptive aspects of inflexible physiological reactivity were tempered for low reappraisers with more moderate levels of physiological flexibility. At high levels of HF-HRV, the association was reversed, such that more flexible physiological reactivity was associated with higher negative affectivity among low reappraisers. As mentioned earlier, excessive physiological flexibility may promote excessive emotional reactivity, which in the absence of emotion regulation strategies that down-regulate the experience of negative emotions, could explain the positive association between HF-HRV and negative affectivity at very high levels of HF-HRV. This excessive reactivity could lead individuals with high HF-HRV to become overly engaged with their social and emotional environments, which can be maladaptive at extreme levels (Kogan et al., 2014). Specifically, individuals with very high HF-HRV may become so involved that they self-attribute blame for the negative emotions of others 
(Ioannou et al., 2013) or engage in overly-dependent interpersonal behaviors, such as excessive reassurance seeking (Joiner \& Metalsky, 2001).

Thus, moderate levels of top-down regulation of sympathetic and parasympathetic influences appear to be the most adaptive in the context of low trait reappraisal and were associated with the lowest levels of negative affectivity. Moderate HF-HRV likely reflects the optimal balance of physiological flexibility, such that the individual is able to track changing environmental demands without being too emotionally reactive. Put another way, these individuals incorporate the relevant emotional cues in their environment, while also disregarding irrelevant information, in order to produce an appropriate physiological response given current contextual demands. If these individuals consistently produce context-appropriate physiological responses, there may be less of a need for them use reappraisal to regulate their emotions and thus they have low negative affectivity despite also having low levels of cognitive reappraisal.

Identification of this quadratic association is important because HF-HRV is typically conceived as having a linear association with negative emotional responding, such that higher HF-HRV unilaterally reflects better physiological and emotion regulation (Appelhans \& Luecken, 2006; Thayer et al., 2009; Spangler et al., 2015). The current results indicate that this is not always the case, and that for certain individuals (i.e., those with low reappraisal tendencies), very high HF-HRV can actually be maladaptive and associated with high negative affectivity. One possible explanation for findings of linear associations between HF-HRV and negative affectivity in previous studies may have to do with the distribution of HF-HRV in study samples. For example, studies that have found a negative linear association between HF-HRV and negative 
affectivity may have oversampled the low-to-moderate range of HF-HRV, thereby capturing an effect that may only be present at low to moderate levels of HF-HRV. These studies have had samples comprised of predominately White participants and thus are expected to have lower levels of HF-HRV than samples that include a larger proportion of Black/African American participants who have been shown to have higher HF-HRV (Liao et al., 1995; Antelmi, De Paula, Shinzato, Peres, Mansur, \& Grupi, 2004). It is not uncommon for both linear and quadratic effects to be significant within the same model (Cohen, Cohen, West, \& Aiken, 2013), meaning that if the researchers did not test quadratic effects, the association between HF-HRV and negative affectivity may be mistakenly interpreted as strictly linear (Spangler et al., 2015). However, even if the researchers tested quadratic effects they still may not have found a quadratic association between HF-HRV and negative affectivity as results of the current study suggest that the link may depend on an individual's trait-based emotion regulation tendencies.

The present findings extend previous research by showing that the association between an individual's physiological reactivity tendencies and negative affectivity differs as a function of their self-reported use of cognitive reappraisal. Specifically, among individuals with high trait cognitive reappraisal, there was no significant linear or quadratic association between HF-HRV and negative affectivity. This lack of association likely reflects the effectiveness of reappraisal at downregulating the subjective experience of negative emotions. By altering the emotional response before it has been fully experienced by the individual, cognitive reappraisal appears to ameliorate the maladaptive aspects of very high and low HF-HRV. For instance, individuals with low HF-HRV may have difficulty producing flexible physiological responses, which can lead 
them to be more anxious; however, if they have high reappraisal tendencies then they may change their interpretation of their situation in such a way that minimizes any potential emotional distress. Likewise, individuals with very high HF-HRV may be overly engaged with their social environments, which can lead to negative outcomes; however if they are modifying the emotional response before it has been fully experienced then they are unlikely to have high negative affectivity. Thus, individuals who frequently use reappraisal to regulate their emotions exhibit low negative affectivity regardless of their HF-HRV. This finding lends support for the idea that our emotion response tendencies are the result of the combined influence of our biological reactivity and behavioral regulation tendencies. Furthermore, this finding opens the door for future research to explore other emotion regulation strategies as possible moderators of the association between HF-HRV and trait affectivity.

It is important to note that at extremely high levels of trait cognitive reappraisal, there was an unexpected negative quadratic association between HF-HRV and negative affectivity, such that very high and low HF-HRV were associated with the lowest levels of negative affectivity. One possible explanation comes from Porges' Polyvagal Theory, which posits that resting HF-HRV promotes social engagement and approach related behaviors (Porges, 2007; Beauchaine, 2001). From this perspective, individuals with low HF-HRV may be disengaged from their environments, meaning they are less likely to attend to and appraise changes in their environment and thus have fewer emotional responses in general, which, if always reappraised, could explain their low negative affectivity. On the other end of the spectrum, the combination of high HF-HRV and extremely high levels of trait reappraisal could lead individuals to be so regulated that 
they are unlikely to experience and identify any negative emotions. Again this is just one possible explanation, and other interpretations are possible as these influences may be bidirectional or be due to the same underlying processes. Though it may sound contradictory at first, I interpret the association between high and low HF-HRV and low negative affectivity among very high reappraisers as reflecting maladaptive regulation processes. According to Butler et al. (2006), adaptive emotion regulation does not consist of experiencing no negative emotions all the time, but is rather the experience of adequate emotions of appropriate intensity given current contextual demands (Geisler et al., 2013). Being able to experience and identify some negative emotions is adaptive (Geisler et al., 2013). Thus, moderate HF-HRV may reflect the most adaptive levels of physiological flexibility among individuals with either low or extremely high levels of trait reappraisal.

\subsection{Limitations}

Several limitations of the study should be noted. Due to the correlational nature of the current study, causal inferences are not possible. This study also relied exclusively on self-report measures of negative affectivity and trait reappraisal. While self-report measures offer an important perspective/component, they are just one measurement of one aspect of these complex and complicated constructs. Future research should utilize multi-method assessments of these complex constructs, such as informant reports or daily ratings, in addition to self-reports to provide stronger corroboration of the findings. Finally, respiration rate and depth, which some researchers suggest are related to resting HF-HRV (Grossman \& Taylor, 2007), were not measured. Some researchers argue that this introduces a potential confound and additional error variance when using resting HF- 
HRV; however, measures of resting HF-HRV that control for respiration are highly correlated with those that do not (Houtveen, Rietveld, \& De Geus, 2002) and there is debate in the literature regarding the necessity of controlling for respiration when assessing resting HF-HRV, as assessments of HF-HRV while at rest have been found to be less susceptible to respiratory confounds than those conducted under mental, emotional, or physical demands (Grossman \& Taylor, 2007; Houtveen et al., 2002).

\subsection{Future Directions}

The findings of the study are consistent with the idea that very high physiological flexibility underlies tendencies to be emotional reactive, which contributes to high negative affectivity among individuals with low trait reappraisal. Future studies that directly measure trait emotional reactivity could assess this postulated mediating mechanism as well as other potential mediators between HF-HRV and affective style, such as executive control or behavioral inhibition.

I have proposed self-attributed blame for others' emotions as one possible explanation for the association between high HF-HRV and high negative affectivity at low levels of trait reappraisal. It will be important for future researchers to examine whether individuals with high HF-HRV and low reappraisal tendencies have high negative affectivity due to tendencies to blame themselves or take responsibility for the emotions of others by testing self-blame as a mediator of this moderated association. Furthermore, future studies would benefit from examining whether the present findings hold at the state level across different social contexts, such as supportive vs. hostile social environments. For example, social environments characterized by social support and positive social feedback could attenuate the association between high HF-HRV and high 
negative affectivity among low reappraiser whereas hostile social environments characterized by social rejection and negative social feedback could potentiate it.

A particularly novel contribution of the current study has been to show that HFHRV's relation to negative affectivity differs as a function of the individual's selfreported use of cognitive reappraisal. A promising avenue for future research would be to examine if other cognitive-related variables, such as acceptance or mindfulness tendencies, affect these processes in similar ways. It is expected that individuals with low acceptance and mindfulness tendencies would exhibit similar associations between high and low HF-HRV and high negative affectivity. Individuals with low HF-HRV and low acceptance and mindfulness tendencies may lack the physiological and cognitive flexibility to shift out of negative emotional states whereas individuals with high HFHRV and low acceptance and mindfulness tendencies could potentially lack the emotional awareness necessary to differentiate between their own emotions and the emotions of others (Hill \& Updegraff, 2012), leading them to be overly engaged with the emotions of others. Future research should also examine whether it is merely the absence of adaptive regulation tendencies that lead individuals with high and low HF-HRV to experience high negative affectivity or if these individuals are also engaging in potentially maladaptive behaviors associated with greater tendencies to experience negative emotions, such as rumination or excessive reassurance seeking. Finally, future studies could also benefit from examining the moderating role of additional emotion regulation strategies that exert their influence at different points in the emotion generation cycle. For example, response-focused regulation strategies such as expressive suppression would likely be less effective at ameliorating the maladaptive aspects of high 
and low HF-HRV as its effect on the emotional response does not occur until after it has been fully formed. Such research could potentially provide targets for treatment based upon individuals' physiological reactivity tendencies.

The current study focused on trait-level biological and behavioral processes predicting an individual's trait-like affective style. An important future direction would be to examine these processes at the state-level. Future studies that experimentally manipulate emotion regulation strategies in the laboratory setting would help elucidate the causality of the associations between HF-HRV, negative affect, and cognitive reappraisal as well as allow researchers to examine how Gross' model of emotion regulation plays out in real time. Furthermore, incorporating neuroimaging techniques would help identify the underlying neural correlates of these processes and provide a more comprehensive and holistic understanding of how the multiple systems subserving emotion and emotion regulation are integrated. This is especially important as very few studies have included measures of HF-HRV and functional brain imaging within the same study (Matthews, Paulus, Simmons, Nelesen, \& Dimsdale, 2004; Neumann et al., 2006; O’Connor, Gundel, McRae, \& Lane, 2007; Mujica-Parodi et al., 2009) and even fewer have measured HF-HRV during the actual imaging task (Lane, McRae, Reiman, Chen, Ahern, \& Thayer, 2009; Critchley et al., 2003; Gianaros, Van der Veen, \& Jennings, 2004). Future studies that included measures of resting HF-HRV, trait affectivity, and trait reappraisal, and also instructed participants to reappraise emotional stimuli while having their brain scanned with functioning imaging technology and their heart rate recorded would allow researchers to distinguish between the state and trait effects of these processes. 


\subsection{Conclusions}

In sum, the findings from the current study suggest that individual differences in tendencies to reappraise potentially emotion-eliciting situations can help explain the mixed findings regarding HF-HRV's association with negative affectivity. Specifically, when individuals do not frequently employ reappraisal to regulate their emotions, excessively high and low HF-HRV are associated with a high tendency to experience negative emotions. Moderate levels of HF-HRV appear most adaptive and were associated with low negative affectivity.

The current study extends the existing literature by providing evidence for the nonlinear aspects of resting HF-HRV as well as for the moderating influence of emotion regulation strategies, such as cognitive reappraisal. Further, the current study adds to a growing body of research suggesting that high HF-HRV may not always be an adaptive characteristic, which is contrary to traditional views (Thayer \& Lane, 2000). The results of the current study also dovetail the findings of Spangler et al. (2015), which concluded that the status of HF-HRV as an adaptive indicator may be subject to the type of emotion regulation strategy employed. A strength of the current study has been the integration of both biological and behavioral models of emotion and emotion regulation. The NIM may focus on the neural and physiological systems governing emotion while Gross' process model focuses on the behavioral processes regulating emotions; however, these processes operate simultaneously and inform one another. Thus, research focusing on just one of these systems may not be comprehensive and could lead researchers to draw incorrect conclusions as they did not account for all of these systems. These findings highlight the complexity of the interactions among the multiple processes underlying our subjective 
experience of emotions and the need for future research to examine how the multiple systems underlying our emotions are integrated. 


\section{REFERENCES}

Antelmi, I., De Paula, R. S., Shinzato, A. R., Peres, C. A., Mansur, A. J., \& Grupi, C. J. (2004). Influence of age, gender, body mass index, and functional capacity on heart rate variability in a cohort of subjects without heart disease. The American journal of cardiology, 93(3), 381-385. doi: 10.1016/j.amjcard.2003.09.065

Appelhans, B. M., \& Luecken, L. J. (2006). Heart rate variability as an index of regulated emotional responding. Review of General Psychology, 10(3), 229-240.

doi:10.1037/1089-2680.10.3.229

Barbas, H., Saha, S., Rempel-Clower, N., \& Ghashghaei, T. (2003). Serial pathways from primate prefrontal cortex to autonomic areas may influence emotional expression. BMC Neuroscience, 4(1), 1-12. doi:10.1186/1471-2202-4-25

Beauchaine, T. P. (2001). Vagal tone, development, and Gray's motivational theory: Toward an integrated model of autonomic nervous system functioning in psychopathology. Development and Psychopathology, 13(2), 183-214. doi:10.1017/S0954579401002012

Beauregard, M., Levesque, J., \& Bourgouin, P. (2001). Neural correlates of conscious self-regulation of emotion. The Journal of Neuroscience. doi: 0270$6474 / 01 / 210001-06 \$ 15.00 / 0$

Belsley, D. A., Kuh, E., \& Welsch, R. E. (1980). Wiley Series in Probability and Statistics. Regression Diagnostics: Identifying Influential Data and Sources of Collinearity, 293-300. doi: 10.1002/0471725153.scard

Berntson, G. G., Thomas Bigger, J., Eckberg, D. L., Grossman, P., Kaufmann, P. G., Malik, M., et al. (1997). Heart rate variability: origins, methods, and interpretive 
caveats. Psychophysiology, 34(6), 623-648. doi: 10.1111/j.1469-

8986.1997.tb02140.x

Bleil, M. E., Gianaros, P. J., Jennings, J. R., Flory, J. D., \& Manuck, S. B. (2008). Trait Negative Affect: Toward an Integrated Model of Understanding Psychological Risk for Impairment in Cardiac Autonomic Function. Psychosomatic Medicine, 70(3), 328-337. doi:10.1097/PSY.0b013e31816baefa

Bollen, K. A., \& Jackman, R. W. (1990). Regression diagnostics: An expository treatment of outliers and influential cases. Modern methods of data analysis, 257291.

Butler, E. A., Wilhelm, F. H., \& Gross, J. J. (2006). Respiratory sinus arrhythmia, emotion, and emotion regulation during social interaction. Psychophysiology, 43(6), 612-622. doi:10.1111/j.1469-8986.2006.00467.x

Carlson, J. M., Dikecligil, G. N., Greenberg, T., \& Mujica-Parodi, L. R. (2012). Trait reappraisal is associated with resilience to acute psychological stress. Journal of Research in Personality, 46(5), 609-613. doi:10.1016/j.jrp.2012.05.003

Cohen, J., Cohen, P., West, S. G., \& Aiken, L. S. (2013). Applied multiple regression/correlation analysis for the behavioral sciences. Routledge.

Cook, R. D., \& Weisberg, S. (1982). Residuals and influence in regression. New York: Chapman and Hall.

Crawford, J. R. and Henry, J. D. (2004), The Positive and Negative Affect Schedule (PANAS): Construct validity, measurement properties and normative data in a large non-clinical sample. British Journal of Clinical Psychology, 43, 245-265. doi: $10.1348 / 0144665031752934$ 
Critchley, H. D., Mathias, C. J., Josephs, O., O’doherty, J., Zanini, S., Dewar, B. K., ... \& Dolan, R. J. (2003). Human cingulate cortex and autonomic control: converging neuroimaging and clinical evidence. Brain, 126(10), 2139-2152. doi: 10.1093/brain/awg216

Cutuli, D. (2014). Cognitive reappraisal and expressive suppression strategies role in the emotion regulation: an overview on their modulatory effects and neural correlates. Frontiers in Systems Neuroscience, 8. doi: 10.3389/fnsys.2014.00175

Dennis, T. A. (2007). Interactions between emotion regulation strategies and affective style: Implications for trait anxiety versus depressed mood. Motivation and Emotion, 31(3), 200-207. doi:10.1007/s11031-007-9069-6

Denson, T. F., Grisham, J. R., \& Moulds, M. L. (2011). Cognitive reappraisal increases heart rate variability in response to an anger provocation. Motivation and Emotion, 35(1), 14-22. doi: 10.1007/s11031-011-9201-5

Di Simplicio, M., Costoloni, G., Western, D., Hanson, B., Taggart, P., \& Harmer, C. J. (2012). Decreased heart rate variability during emotion regulation in subjects at risk for psychopathology. Psychological Medicine, 42(8), 1775-83.

doi:http://dx.doi.org/10.1017/S0033291711002479

Drabant, E. M., McRae, K., Manuck, S. B., Hariri, A. R., \& Gross, J. J. (2009). Individual differences in typical reappraisal use predict amygdala and prefrontal responses. Biological psychiatry, 65(5), 367-373. doi:

10.1016/j.biopsych.2008.09.007 
Faul, F., Erdfelder, E., Lang, A.-G., \& Buchner, A. (2007) G*Power 3: A flexible statistical power analysis program for the social, behavioral, and biomedical sciences. Behavior Research Methods, 39(2), 175-191. doi:10.3758/bf03193146

Fredrickson, B. L., Tugade, M. M., Waugh, C. E., \& Larkin, G. R. (2003). What good are positive emotions in crisis? A prospective study of resilience and emotions following the terrorist attacks on the United States on September 11th, 2001. Journal of Personality and Social Psychology, 84(2), 365-376. doi:10.1037/00223514.84 .2 .365

Friedman, B. H. (2007). An autonomic flexibility-neurovisceral integration model of anxiety and cardiac vagal tone. Biological Psychology, 74(2), 185-199. doi:http://dx.doi.org/10.1016/j.biopsycho.2005.08.009

Friedman, B. H., \& Thayer, J. F. (1998). Autonomic balance revisited: panic anxiety and heart rate variability. Journal of Psychosomatic Research, 44(1), 133-151. doi: 10.1016/S0022-3999(97)00202-X

Geisler, F. C., Kubiak, T., Siewert, K., \& Weber, H. (2013). Cardiac vagal tone is associated with social engagement and self-regulation. Biological psychology, 93(2), 279-286. doi:10.1016/j.biopsycho.2013.02.013

Gianaros, P. J., Van der Veen, F. M., \& Jennings, J. R. (2004). Regional cerebral blood flow correlates with heart period and high-frequency heart period variability during working-memory tasks: Implications for the cortical and subcortical regulation of cardiac autonomic activity. Psychophysiology, 41(4), 521-530. doi: 10.1111/1469-8986.2004.00179.x 
Goldin, P. R., McRae, K., Ramel, W., \& Gross, J. J. (2008). The neural bases of emotion regulation: Reappraisal and suppression of negative emotion. Biological Psychiatry, 63(6), 577-586. doi:10.1016/j.biopsych.2007.05.031

Gross, J. J. (1998a). Antecedent- and response-focused emotion regulation: Divergent consequences for experience, expression, and physiology. Journal of Personality and Social Psychology, 74(1), 224-237. doi:10.1037/0022-3514.74.1.224

Gross, J. J. (1998b). The emerging field of emotion regulation: An integrative review. Review Of General Psychology, 2(3), 271-299. doi:10.1037/1089-2680.2.3.271

Gross, J. J. (1999). Emotion regulation: Past, present, future. Cognition and Emotion, 13(5), 551-573. doi:10.1080/026999399379186

Gross, J. J. (2002). Emotion regulation: Affective, cognitive, and social consequences. Psychophysiology, 39(3), 281-291. doi: 10.1017/S0048577201393198.

Gross, J. J. (2014). Handbook of emotion regulation (2nd ed.). New York, NY, US: Guilford Press.

Gross, J. J., \& John, O. P. (2003). Individual differences in two emotion regulation processes: Implications for affect, relationships, and well-being. Journal of Personality and Social Psychology, 85(2), 348-362. doi:10.1037/00223514.85 .2 .348

Grossman, P., \& Taylor, E. W. (2007). Toward understanding respiratory sinus arrhythmia: relations to cardiac vagal tone, evolution and biobehavioral functions. Biological psychology, 74(2), 263-285. doi:

10.1016/j.biopsycho.2005.11.014 
Healy, B., Treadwell, A., \& Reagan, M. (2011). Measures of RSA suppression, attentional control, and negative affect predict self-ratings of executive functions. Journal of Psychophysiology, 25, 164-173. doi: 10.1027/0269-8803/a000053

Hill, C. L., \& Updegraff, J. A. (2012). Mindfulness and its relationship to emotional regulation. Emotion, 12(1), 81. doi: 10.1037/a0026355

Houtveen, J. H., Rietveld, S., \& Geus, E. J. (2002). Contribution of tonic vagal modulation of heart rate, central respiratory drive, respiratory depth, and respiratory frequency to respiratory sinus arrhythmia during mental stress and physical exercise. Psychophysiology, 39(4), 427-436. doi: 10.1111/14698986.3940427

Hughes, J. W., \& Stoney, C. M. (2000). Depressed mood is related to high-frequency heart rate variability during stressors. Psychosomatic Medicine, 62(6), 796-803.

Ingjaldsson, J. T., Laberg, J. C., \& Thayer, J. F. (2003). Reduced heart rate variability in chronic alcohol abuse: Relationship with negative mood, chronic thought suppression, and compulsive drinking. Biological Psychiatry, 54(12), 1427-1436. doi:10.1016/S0006-3223(02)01926-1

Ioannou, S., Ebisch, S., Aureli, T., Bafunno, D., Ioannides, H. A., Cardone, D., Manini, B., Romani, G. L., Gallese, V., \& Merla, A. (2013). The autonomic signature of guilt in children: A thermal infrared imaging study, PloS one, 8(11), e79440. doi: 10.1371/journal.pone.0079440

Jaccard, J., \& Turrisi, R. (2003). Interaction effects in multiple regression (No. 72). Sage Publications. 
Jackson, D. C., Malmstadt, J. R., Larson, C. L., \& Davidson, R. J. (2000). Suppression and enhancement of emotional responses to unpleasant pictures. Psychophysiology, 37(4), 515-522. doi: 10.1111/1469-8986.3740515

Johnsen, B. H., Thayer, J. F., Laberg, J. C., Wormnes, B., Raadal, M., Skaret, E., Kvale, G., \& Berg, E. (2003). Attentional and physiological characteristics of patients with dental anxiety. Journal of Anxiety Disorders, 17(1), 75-87. doi:10.1016/S0887-6185(02)00178-0

Joiner, T. E., \& Metalsky, G. I. (2001). Excessive reassurance seeking: Delineating a risk factor involved in the development of depressive symptoms. Psychological Science, 12(5), 371-378. doi:10.1111/1467-9280.00369

Kettunen, J., Ravaja, N., Näätänen, P., \& Keltikangas-Järvinen, L. (2000). The relationship of respiratory sinus arrhythmia to the co-activation of autonomic and facial responses during the Rorschach test. Psychophysiology, 37(2), 242-250. doi:10.1017/S0048577200982088

Kleiger, R. E., Stein, P. K., \& Bigger, J. T. (2005). Heart rate variability: measurement and clinical utility. Annals of Noninvasive Electrocardiology, 10(1), 88-101. doi: 10.1111/j.1542-474X.2005.10101.x

Kogan, A., Gruber, J., Shallcross, A. J., Ford, B. Q., \& Mauss, I. B. (2013). Too much of a good thing? Cardiac vagal tone's nonlinear relationship with well-being. Emotion, 13(4), 599-604. doi:10.1037/a0032725

Kogan, A., Oveis, C., Carr, E. W., Gruber, J., Mauss, I. B., Shallcross, A., \& ... Keltner, D. (2014). Vagal activity is quadratically related to prosocial traits, prosocial 
emotions, and observer perceptions of prosociality. Journal of Personality and Social Psychology, 107(6), 1051-1063. doi:10.1037/a0037509

Kok, B. E., \& Fredrickson, B. L. (2010). Upward spirals of the heart: Autonomic flexibility, as indexed by vagal tone, reciprocally and prospectively predicts positive emotions and social connectedness. Biological Psychology, 85(3), 432436. doi:10.1016/j.biopsycho.2010.09.005

Lane, R. D., McRae, K., Reiman, E. M., Chen, K., Ahern, G. L., \& Thayer, J. F. (2009). Neural correlates of heart rate variability during emotion. Neuroimage, 44(1), 213-222. doi: 10.1016/j.neuroimage.2008.07.056

Lehofer, M., Moser, M., Hoehn-Saric, R., Mcleod, D., Liebmann, P., Drnovsek, B., \& ... Zapotoczky, H. (1997). Major depression and cardiac autonomic control. Biological Psychiatry, 42(10), 914-919. doi:10.1016/S0006-3223(96)00494-5

Levenson, R. W. (2003). Blood, Sweat, and Fears: The Autonomic Architecture of Emotion. In P. Ekman, J. J. Campos, R. J. Davidson, F. M. de Waal, P. Ekman, J. J. Campos, ... F. M. de Waal (Eds.), Emotions inside out: 130 years after Darwin's: The expression of the emotions in man and animals (pp. 348-366). New York, NY, US: New York Academy of Sciences.

Liao, D., Barnes, R. W., Chambless, L. E., Simpson, R. J., Sorlie, P., Heiss, G., \& ARIC investigators. (1995). Age, race, and sex differences in autonomic cardiac function measured by spectral analysis of heart rate variability — the ARIC study. The American journal of cardiology, 76(12), 906-912. doi:10.1016/S00029149(99)80260-4 
Lieberman, M. D., Inagaki, T. K., Tabibnia, G., \& Crockett, M. J. (2011). Subjective responses to emotional stimuli during labeling, reappraisal, and distraction. Emotion, 11(3), 468-480. doi:10.1037/a0023503

Malliani, A., Pagani, M., Lombardi, F., \& Cerutti, S. (1991). Cardiovascular neural regulation explored in the frequency domain. Circulation, 84(2), 482-492. doi: 10.1161/01.CIR.84.2.482

Matthews, S. C., Paulus, M. P., Simmons, A. N., Nelesen, R. A., \& Dimsdale, J. E. (2004). Functional subdivisions within anterior cingulate cortex and their relationship to autonomic nervous system function. Neuroimage, 22(3), 11511156. doi: 10.1016/j.neuroimage.2004.03.005

Mor, N., \& Winquist, J. (2002). Self-focused attention and negative affect: A metaanalysis. Psychological Bulletin, 128(4), 638-662. doi:10.1037/00332909.128.4.638

Mujica-Parodi, L. R., Korgaonkar, M., Ravindranath, B., Greenberg, T., Tomasi, D., Wagshul, M., ... \& Chon, K. (2009). Limbic dysregulation is associated with lowered heart rate variability and increased trait anxiety in healthy adults. Human brain mapping, 30(1), 47-58. doi: 10.1002/hbm.20483

Neumann, S. A., Brown, S. M., Ferrell, R. E., Flory, J. D., Manuck, S. B., \& Hariri, A. R. (2006). Human choline transporter gene variation is associated with corticolimbic reactivity and autonomic-cholinergic function. Biological psychiatry, 60(10), 1155-1162. doi: 10.1016/j.biopsych.2006.03.059 
Ochsner, K. N., Bunge, S. A., Gross, J. J., \& Gabrieli, J. E. (2002). Rethinking feelings: An fMRI study of the cognitive regulation of emotion. Journal of Cognitive Neuroscience, 14(8), 1215-1229. doi:10.1162/089892902760807212

Ochsner, K. N., Ray, R. D., Cooper, J. C., Robertson, E. R., Chopra, S., Gabrieli, J. D., \& Gross, J. J. (2004). For better or for worse: neural systems supporting the cognitive down-and up-regulation of negative emotion. Neuroimage, 23(2), 483499. doi: 10.1016/j.neuroimage.2004.06.030

Ochsner, K. N., \& Gross, J. J. (2005). The cognitive control of emotion. Trends in Cognitive Sciences, 9(5), 242-249. doi:10.1016/j.tics.2005.03.010

Ochsner, K. N., \& Gross, J. J. (2008). Cognitive emotion regulation: Insights from social cognitive and affective neuroscience. Current Directions in Psychological Science, 17(2), 153-158. doi:10.1111/j.1467-8721.2008.00566.x

Ochsner, K. N. \& Gross, J. J. (2014). The Neural Bases of Emotion and Emotion Regulation: A Valuation Perspective. In J. Gross (Ed.), Handbook of emotion regulation (pp. 23-43). New York, NY, US: Guilford Press.

O'Connor, M. F., Gündel, H., McRae, K., \& Lane, R. D. (2007). Baseline vagal tone predicts BOLD response during elicitation of grief. Neuropsychopharmacology, 32(10), 2184-2189. doi: 10.1038/sj.npp.1301342

Ode, S., Hilmert, C. J., Zielke, D. J., \& Robinson, M. D. (2010). Neuroticism's importance in understanding the daily life correlates of heart rate variability. Emotion, 10(4), 536-543. doi:10.1037/a0018698 
Oveis, C., Cohen, A. B., Gruber, J., Shiota, M. N., Haidt, J., \& Keltner, D. (2009).

Resting respiratory sinus arrhythmia is associated with tonic positive emotionality. Emotion, 9(2), 265-270. doi:10.1037/a0015383

Porges, S. W. (2007). The polyvagal perspective. Biological Psychology, 74(2), 116-143. doi:10.1016/j.biopsycho.2006.06.009

Porges, S. W., Doussard-Roosevelt, J. A., \& Maita, A. K. (1994). Vagal tone and the physiological regulation of emotion. Monographs of the Society for Research in Child Development, 59(2-3), 167-186. doi:10.2307/1166144

Ray, R. D., McRae, K., Ochsner, K. N., \& Gross, J. J. (2010). Cognitive reappraisal of negative affect: Converging evidence from EMG and self-report. Emotion, 10(4), 587-592. doi:10.1037/a0019015

Riese, H., Rosmalen, J. M., Ormel, J., Van Roon, A., Oldehinkel, A. J., \& Rijsdijk, F. V. (2007). The genetic relationship between neuroticism and autonomic function in female twins. Psychological Medicine, 37(2), 257-267.

doi:10.1017/S0033291706009160

Rottenberg, J. (2007). Cardiac vagal control in depression: A critical analysis. Biological Psychology, 74(2), 200-211. doi:10.1016/j.biopsycho.2005.08.010

Rottenberg, J., Wilhelm, F. H., Gross, J. J., \& Gotlib, I. H. (2002). Respiratory sinus arrhythmia as a predictor of outcome in major depressive disorder. Journal of affective disorders, 71(1), 265-272. doi: 10.1016/S0165-0327(01)00406-2

Ruiz-Padial, E., Sollers, J. I., Vila, J., \& Thayer, J. F. (2003). The rhythm of the heart in the blink of an eye: Emotion-modulated startle magnitude covaries with heart rate variability. Psychophysiology, 40(2), 306-313. doi:10.1111/1469-8986.00032 
Saul, J. P. (1990). Beat-to-beat variations of heart rate reflect modulation of cardiac autonomic outflow. Physiology, 5(1), 32-37.

Schwerdtfeger, A. R., \& Scheel, S. (2012). Self-esteem fluctuations and cardiac vagal control in everyday life. International Journal of Psychophysiology, 83(3), 328335. doi:10.1016/j.ijpsycho.2011.11.016

Shekar, A., Sajdyk, T. J., Gehlert, D. R. \& Rainnie, D. G. (2003), The amygdala, panic disorder, and cardiovascular responses. Annals of the New York Academy of Sciences, 985, 308-325. doi: 10.1111/j.1749-6632.2003.tb07090.x

Shepherd, D., Mulgrew, J., \& Hautus, M. J. (2015). Exploring the autonomic correlates of personality. Autonomic Neuroscience, 193, 127-131. doi:10.1016/j.autneu.2015.05.004

Silvia, P. J., Jackson, B. A., \& Sopko, R. S. (2014). Does baseline heart rate variability reflect stable positive emotionality?. Personality and Individual Differences, 70183-187. doi:10.1016/j.paid.2014.07.003

Spangler, D. P., Bell, M. A., \& Deater-Deckard, K. (2015). Emotion suppression moderates the quadratic association between RSA and executive function. Psychophysiology, 52(9), 1175-1185. doi:10.1111/psyp.12451

Stevens, J. (2002). Applied Multivariate Statistics for the Social Sciences. Mahwah, N.J.: Lawrence Erlbaum Associates, Inc.

Suess, P. E., Porges, S. W., \& Plude, D. J. (1994). Cardiac vagal tone and sustained attention in school-age children. Psychophysiology, 31(1), 17-22. doi:10.1111/j.1469-8986.1994.tb01020.x 
Szasz, P. L., Szentagotai, A., \& Hofmann, S. G. (2011). The effect of emotion regulation strategies on anger. Behaviour Research and Therapy, 49(2), 114-119. doi:10.1016/j.brat.2010.11.011

Tabachnick, B. G., \& Fidell, L. S. (2013). Using multivariate statistics ( $6^{\text {th }}$ Ed.). New Jersey: Pearson.

Task Force of the European Society of Cardiology, \& Task Force of the European Society of Cardiology. (1996). the North American Society of Pacing and Electrophysiology. Heart rate variability: standards of measurement, physiological interpretation and clinical use. Circulation, 93(5), 1043-1065.

Thayer, J. F., Friedman, B. H., \& Borkovec, T. D. (1996). Autonomic characteristics of generalized anxiety disorder and worry. Biological Psychiatry, 39(4), 255-266. doi:10.1016/0006-3223(95)00136-0

Thayer, J. F., \& Lane, R. D. (2000). A model of neurovisceral integration in emotion regulation and dysregulation. Journal Of Affective Disorders, 61(3), 201-216. doi:10.1016/S0165-0327(00)00338-4

Thayer, J. F., \& Lane, R. D. (2002). Perseverative thinking and health: Neurovisceral concomitants. Psychology \& Health, 17(5), 685-695. doi: $10.1080 / 08870440290025867$

Thayer, J. F., \& Lane, R. D. (2009). Claude Bernard and the heart-brain connection: Further elaboration of a model of neurovisceral integration. Neuroscience and Biobehavioral Reviews, 33(2), 81-88. doi:10.1016/j.neubiorev.2008.08.004 
Thayer, J. F., \& Siegle, G. J. (2002). Neurovisceral integration in cardiac and emotional regulation. Engineering in Medicine and Biology Magazine, IEEE, 21(4), 24-29. doi: 10.1109/MEMB.2002.1032635

Thomsen, D. K. (2006). The association between rumination and negative affect: A review. Cognition and Emotion, 20(8), 1216-1235.

doi:10.1080/02699930500473533

Tulen, J. M., Bruijn, J. A., de Man, K. J., van der Velden, E., Pepplinkhuizen, L., \& Veld, A. '. (1996). Anxiety and autonomic regulation in major depressive disorder: An exploratory study. Journal Of Affective Disorders, 40(1-2), 61-71. doi:10.1016/0165-0327(96)00042-0

Wang, Z., Lü, W., \& Qin, R. (2013). Respiratory sinus arrhythmia is associated with trait positive affect and positive emotional expressivity. Biological Psychology, 93(1), 190-196. doi:10.1016/j.biopsycho.2012.12.006

Wang, L., Shi, Z., \& Li, H. (2009). Neuroticism, extraversion, emotion regulation, negative affect and positive affect: The mediating roles of reappraisal and suppression. Social Behavior And Personality, 37(2), 193-194. doi:10.2224/sbp.2009.37.2.193

Wang, X., Thayer, J. F., Treiber, F., \& Snieder, H. (2005). Ethnic differences and heritability of heart rate variability in African-and European American youth. The American journal of cardiology, 96(8), 1166-1172. doi:

10.1016/j.amjcard.2005.06.050

Watkins, L. L., Grossman, P., Krishnan, R., \& Sherwood, A. (1998). Anxiety and vagal control of heart rate. Psychosomatic Medicine, 60(4), 498-502. 
Watson, D., Clark, L. A., \& Tellegen, A. (1988). Development and validation of brief measures of positive and negative affect: The PANAS scales. Journal of Personality and Social Psychology, 54(6), 1063-1070. doi:10.1037/00223514.54.6.1063

Watson, D. C., \& Clark, A. LA (1994). The PANAS-X: Manual for the Positive and Negative Affect Schedule-Expanded Form.

Wolgast, M., Lundh, L., \& Viborg, G. (2011). Cognitive reappraisal and acceptance: An experimental comparison of two emotion regulation strategies. Behaviour Research and Therapy, 49(12), 858-866. doi:10.1016/j.brat.2011.09.011

Woody, M. L., McGeary, J. E., \& Gibb, B. E. (2014). Brooding rumination and heart rate variability in women at high and low risk for depression: Group differences and moderation by COMT genotype. Journal of Abnormal Psychology, 123(1), 61-67. doi:10.1037/a0035450

Yeragani, V. K., Pohl, R., Balon, R., Ramesh, C., Glitz, D., Jung, I., \& Sherwood, P. (1991). Heart rate variability in patients with major depression. Psychiatry Research, 37(1), 35-46. doi:10.1016/0165-1781(91)90104-W

Zahn, D., Adams, J., Krohn, J., Wenzel, M., Mann, C. G., Gomille, L. K., \& ... Kubiak, T. (2016). Heart rate variability and self-control—A meta-analysis. Biological Psychology, 1159-26. doi:10.1016/j.biopsycho.2015.12.007 